\title{
Adsorption of Protein on a Au Surface Studied by All-Atom Atomistic Simulations
}

\author{
By \\ Aoran Wei \\ A thesis submitted to the Faculty of Graduate Studies of
The University of Manitoba
in partial fulfilment of the requirements for the degree of
}

MASTER OF SCIENCE

Department of Mechanical Engineering

Faculty of Graduate Study

University of Manitoba

Winnipeg, Manitoba

August 2016

Copyright @ 2016 by Aoran Wei 


\begin{abstract}
In this work, the adsorption of protein on Au surface coated by self-assembled monolayers (SAMs) of alkanethiol chains is studied by molecular dynamics simulations with an all-atom model. Particularly, a more realistic embedded-atom method potential has been used to characterize the $\mathrm{Au}-\mathrm{Au}$ interactions in the system as compared to previous studies. With this all-atom model, many experimental observations have been reproduced from the simulations. It is found that the SAMs have the lowest adsorption energy on $\mathrm{Au}$ (111) surface where the alkanethiol chains form a well-ordered $(\sqrt{ } 3 \mathrm{x} \sqrt{ } 3) \mathrm{R} 30^{\circ}$ triangular lattice at $300 \mathrm{~K}$. Furthermore, it is confirmed that carboxyl-terminated SAMs are more effective to absorb proteins than the methyl-terminated SAMs. Base on the simulation results, we propose that the experimentally observed aggregation of protein-Au nanoparticle conjugates is mainly due to the electrostatic interactions between protein amino acids and carboxyl-terminated SAMs from multiple Au surfaces.
\end{abstract}




\section{ACKNOWLEDGMENTS}

I would like to take this opportunity to thank my advisor, Dr. Chuang Deng, whose constant support and encouragement has facilitated my research and this thesis. He has enriched knowledge of materials science and was always very patient when we solved research problems together. Dr. Deng gave me the opportunity to study abroad in Canada, helping me build my personality and broadening my horizons to the world. I will always be grateful.

In addition, I would like to express my gratitude and appreciation to my committee members, Dr. Malcolm Xing form the Department of Mechanical Engineering and Dr. Peter Budzelaar from the Department of Chemistry. Thank you for reviewing my thesis and attending my defense. And I would also like to give my thanks to my colleagues in my lab and friends who helped and supported me all the time.

Finally, I would like to thank my family, especially my mother. They are the most important people to me in my life. And wish my grandmother could be healthy all the time. Without their support and understanding, it is impossible for me to obtain this degree. 


\section{LIST OF ABBREVIATION}

AuNP

SAM

FF

EAM

PBC

Lyz

MD simulation

TEM

LAMMPS

NP

IR spectroscopy

AuNC

BSA

CD

CHARMM

OPLS

MC

NVT

NPT

PDB
Au nanoparticle

Self-assembled monolayer

Force field

Embedded atom model potential

Periodic boundary conditions

Lysozyme

Molecular dynamics simulation

Transmission electron microscopy

Large-scale atomic/molecular massively

parallel simulator

Nanoparticle

Infrared spectroscopy

Au nanocage

Bovine serum albumin

Circular dichroism

Chemistry at Harvard macromolecular

mechanics

Optimized potential for liquid simulations

Monte Carlo

Canonical ensemble

Isothermal-isobaric ensemble

Protein data bank 


\section{TABLE OF CONTENTS}

CHAPTER 1 MOTIVATION AND OUTLINE OF THE THESIS .................................... 1

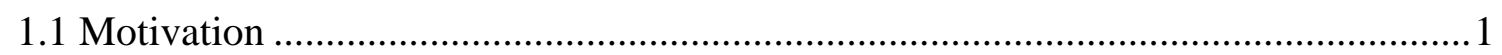

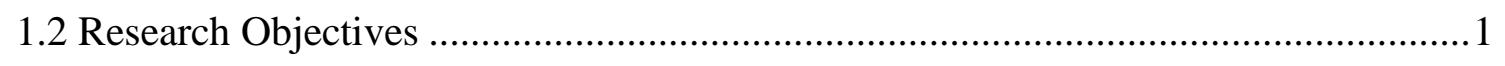

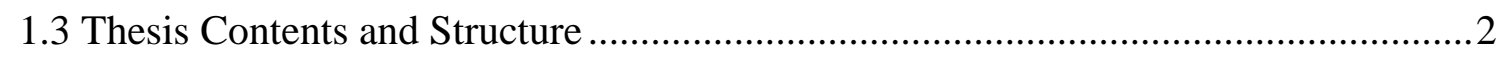

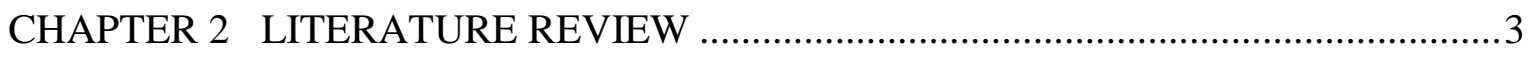

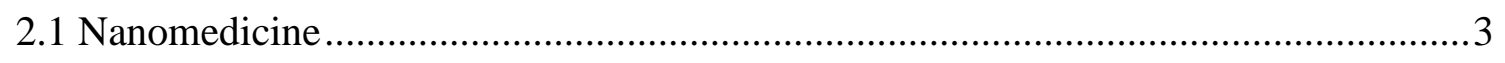

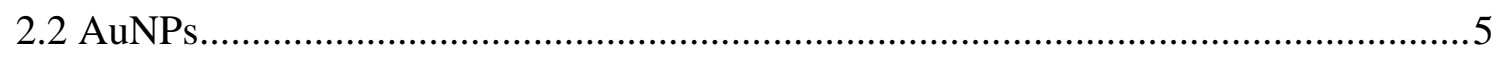

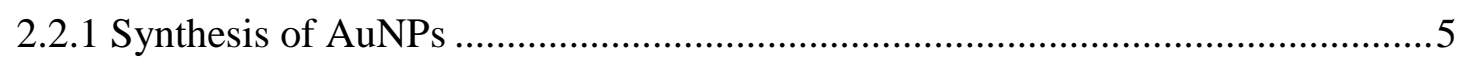

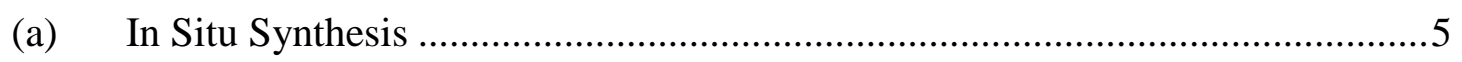

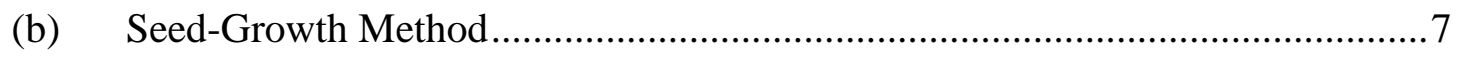

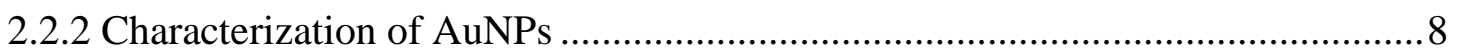

(a) Transmission Electron Microscopy (TEM) ...................................................... 8

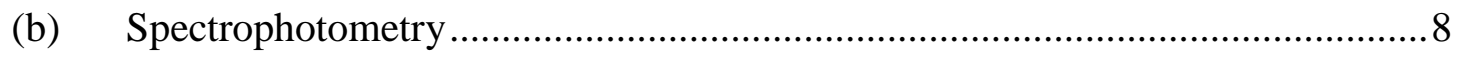

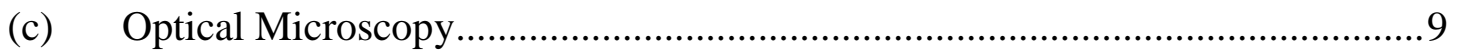

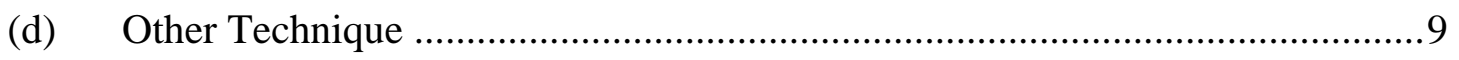

2.2.3 Self-Assembled Monolayers (SAMs) on AuNPs ............................................... 10

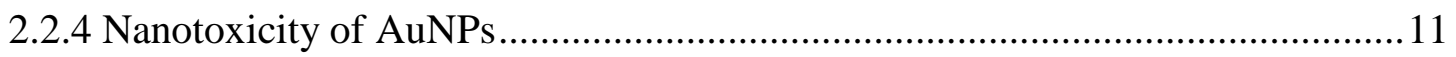

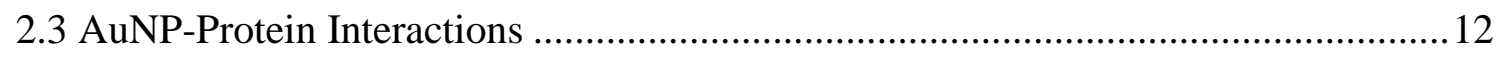

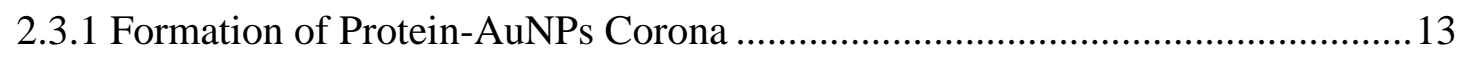

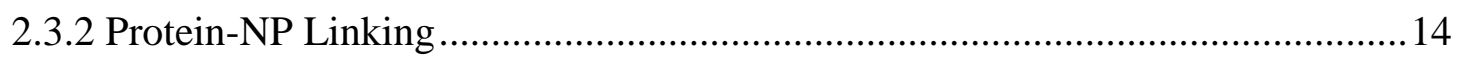

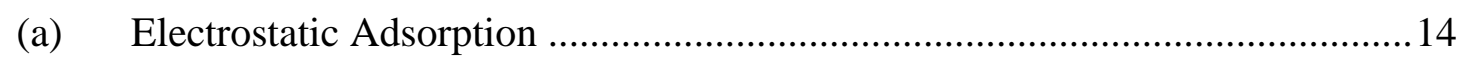

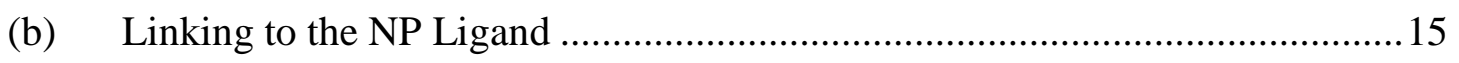

(c) Linking Using Specific Affinity of Protein for a Cofactor .............................15

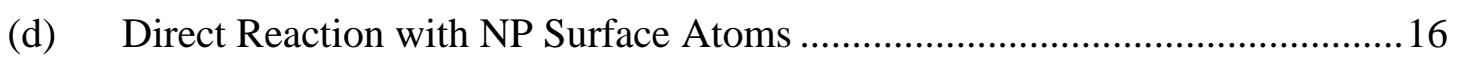

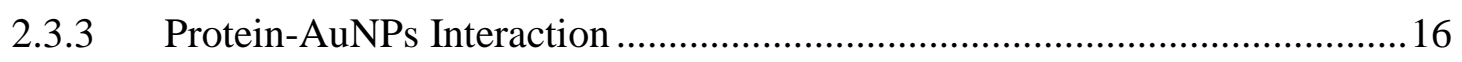

(a) The Influences of AuNP Size, Shape, Surface on the Protein Adsorption ........16

(b) Conformational Changes of Proteins Upon Binding to NPs. ...........................19

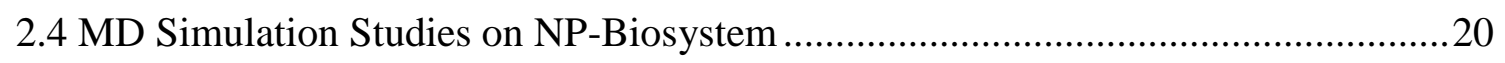

2.4.1 Past MD Studies on AuNP-Biosystem Interactions ............................................21

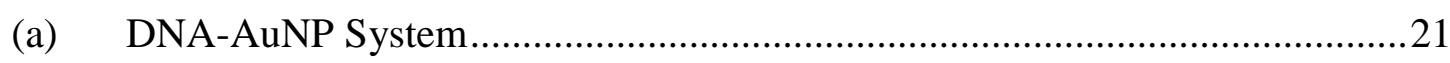

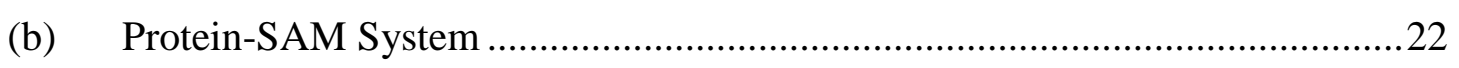




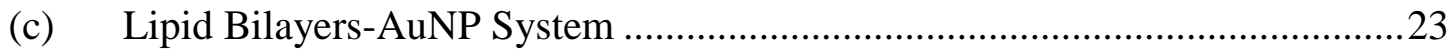

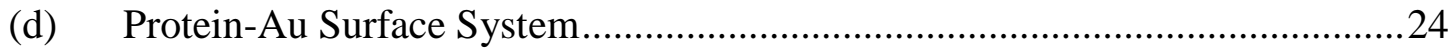

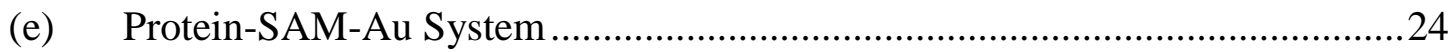

2.4.2 Force Field (FF) for Protein-AuNP Simulation Studies .............................25

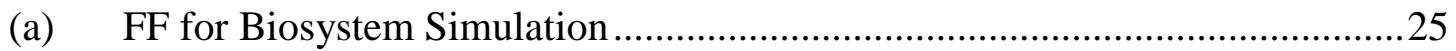

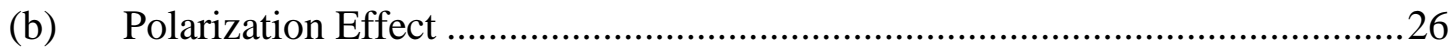

(c) FF Used for Metal-Protein Interactions ......................................................2

(d) Embedded-Atom Method (EAM) for AuNPs..................................................28

2.4.3 Challenges of Nano-Bio System Studies by MD Simulation ............................29

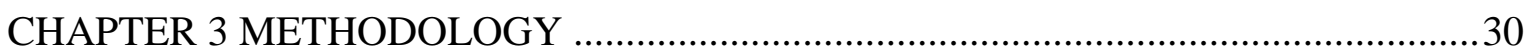

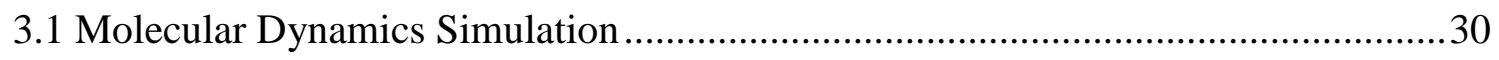

3.1.1 The Advantages of Molecular Dynamics ......................................................... 31

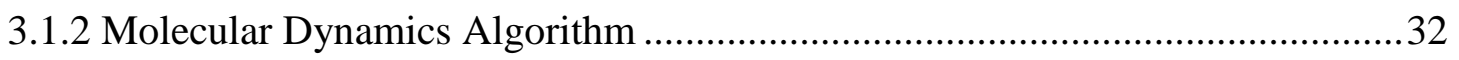

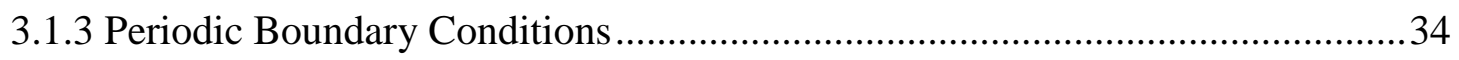

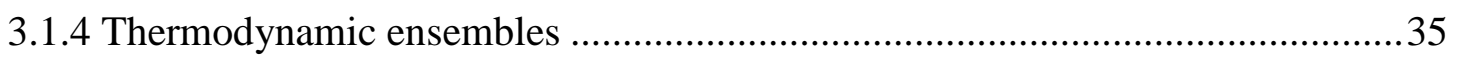

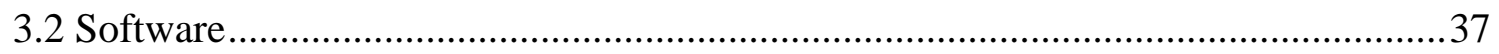

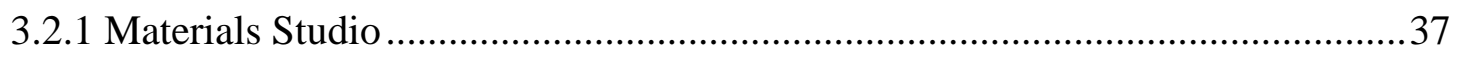

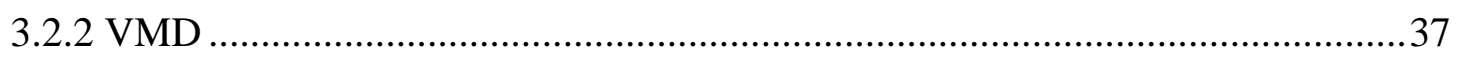

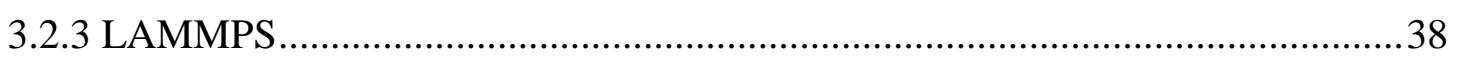

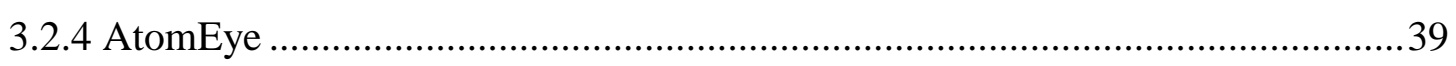

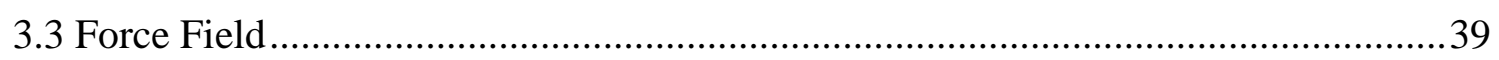

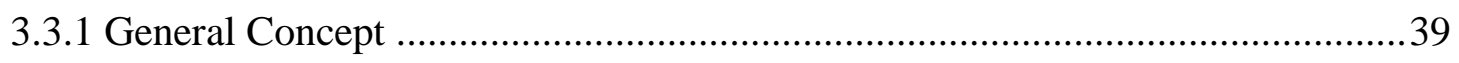

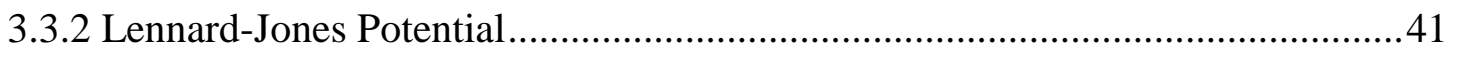

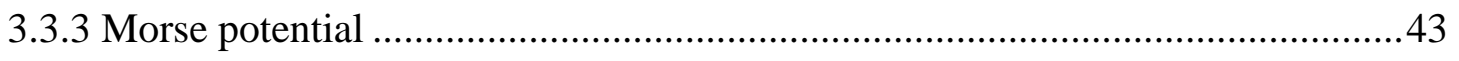

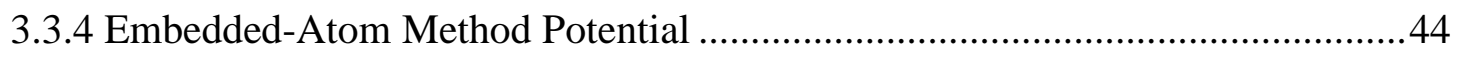

3.4 Model Construction and Simulation Settings ...................................................... 46

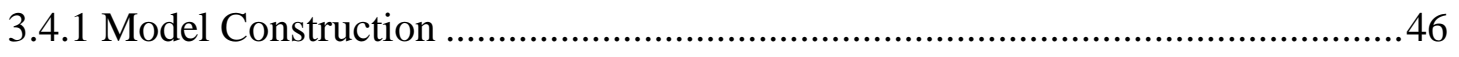

3.4.2 System Geometry and Simulation Settings ................................................... 47

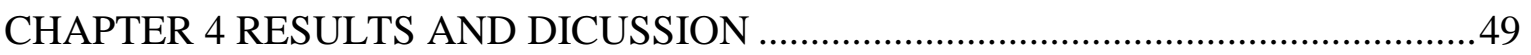

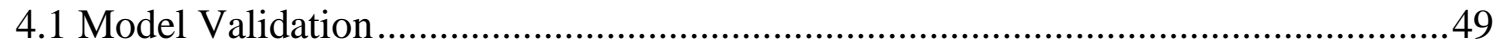

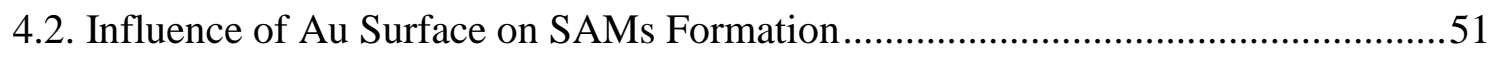

4.3. Influence of Alkanethiol Chain Length on SAMs Formation ..................................54

4.4 Influence of Temperature on SAMs Arrangement on Spherical AuNPs ...................56

4.5 Influences of SAMs Terminal Functional Group on Protein Adsorption ..................57

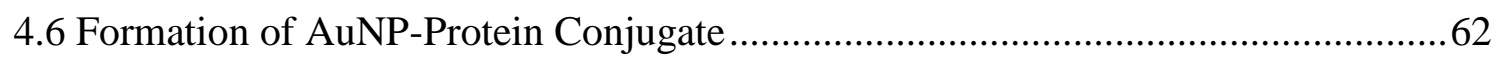




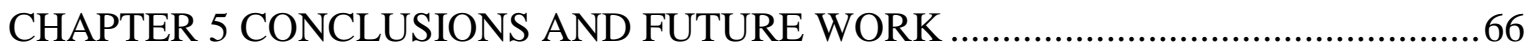

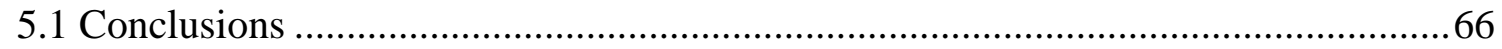

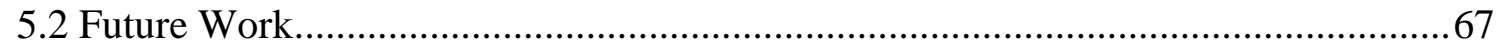

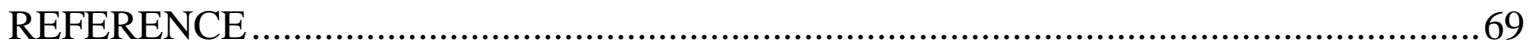




\section{TABLE OF FIGURES}

Figure 2-1. Nanomedicine publications and patents worldwide ${ }^{1}$

Figure 2-2. TEM image of larger gold particles prepared from seed: (a) 5.5 \pm 0.6 , (b)

$8.0 \pm 0.8$, (c) $17 \pm 2.5$, and (d) $37 \pm 5 \mathrm{~nm}$ after separation of rods $\mathrm{s}^{29}$ 8

Figure 2-3. Protein corona on $\mathrm{NPs}^{53}$. 13

Figure 2-4. NP-protein labeling strategies: (a) electrostatic attachment of protein, (b) covalent attachment to the NP ligand, (c) attachment of a protein cofactor on NP, and (d) direct linkage of amino acid on the NP core

Figure 2-5. Structure of DNA-AuNP and snapshots during simulation. .22

Figure 2-6. Final snapshot of the three peptide/SAMs system. .23

Figure 2-7. Snapshot of the interaction between the metal nanoparticles and the membrane

Figure 2-8. Final adsorbed conformation of 6NGBP on Au (001) from MD simulations in vacuum at $310 \mathrm{~K}$. The tube represents the backbone of the protein. Adsorbed sections are shown in blue. The texture of the Au surface [in yellow] is omitted from the top views for clarity in the first figure ${ }^{97}$. .24

Figure 2-9. Cyt-c configurations on Au surface covered with carboxyl-terminated SAMs.

The SAMs with increasing dissociation degrees of the terminal carboxyl groups have been

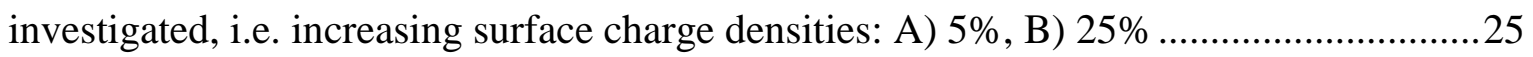

Figure 2-10. Models of polarizable Au surfaces ${ }^{106}$ .28

Figure 3-1, Simulations as a bridge between microscopic and macroscopic (left); (b) theory and experiment (right) ${ }^{119}$ .31

Figure 3-2: Schematic representation of the Periodic Boundary Conditions (PBC) ${ }^{122}$.......35

Figure 3-3. Materials Studio software Interface .37

Figure 3-4. VMD software Interface .38 
Figure 3-5, the strength versus distance for the 12-6 Lennard-Jones potential 42

Figure 3-6, the Morse potential (blue) and harmonic oscillator potential (green) .44

Figure 3-7. The pair potential and electron-density function ${ }^{111}$ .45

Figure 3-8. The embedding energy function ${ }^{111}$ 45

Figure 3-9. Atomistic configurations of an assembly of a spherical AuNP with two carboxyl-terminated SAMs. (a) The initial configuration; (b) the configuration after relaxation at $300 \mathrm{~K}$ for $100 \mathrm{ps}$; (c) the configuration after relaxation of additional $180 \mathrm{ps}$ at $1000 \mathrm{~K}$

Figure 4-1. Equilibrated structure of SAMs on Au (111) surface from our MD simulations with one single alkanethiol chain at (a) $300 \mathrm{~K}$ and (b) $600 \mathrm{~K}$ and multiple alkanethiol chains at (c) $300 \mathrm{~K}$ and (d) $600 \mathrm{~K}$, respectively. .50

Figure 4-2, The adsorption of $\mathrm{CH}_{3}$-SAMs on spherical AuNPs at $300 \mathrm{~K}$. .51

Figure 4-3. Side view of the equilibrium SAMs structures on (a) Au (111), (c) Au (110) and (e) $\mathrm{Au}(100)$ at $300 \mathrm{~K}$ and schematic diagram of $\mathrm{S}$ atoms on (b) $\mathrm{Au}$ (111), (d) Au (110) and (f) $\mathrm{Au}(100)$ at $300 \mathrm{~K} . \mathrm{S}$ atoms are changed in (b), (d) and (f) to red color for better view.

Figure 4-4. (a) The energy of a single alkanethiol chain on (100) Au surface before (black line) and after (red line) the adsorption. (b) The adsorption energy of a single alkanethiol chain on $\mathrm{Au}$ (111), (110) and (100) surfaces. .53

Figure 4-5. The influence of chain length on SAMs formation on Au (111) surface at $300 \mathrm{~K}$ .55

Figure 4-6. Snapshots of SAMs on spherical AuNP surfaces at different temperatures at (a) $300 \mathrm{~K}$, (b) $600 \mathrm{~K}$, (c) $900 \mathrm{~K}$ .56

Figure 4-7. Snapshot of the interaction between protein and methyl-terminated SAMs...58 
Figure 4-8. The electrostatic interactions occurred between Carboxyl-terminated SAMs and protein amino acids.

Figure 4-9. (a) and (b) The interaction between two Lysozyme protein molecules. (c) and (d) The interaction between two Au (111) surfaces covered with carboxyl-terminated SAMs.

Figure 4-10. (a) and (b) The adsorption between Lyz protein and two Au surfaces covered with carboxyl-terminated SAMs. (c) and (d) Schematic of Lyz-AuNP conjugate formation proposed in this study ${ }^{134}$

Figure 4-11. (a) and (b) The interaction between two carboxyl-terminated SAMs covered AuNPs. (c) and (d) The interaction between two spherical AuNPs covered with carboxylterminated SAMs and the protein. .64 


\section{CHAPTER 1 MOTIVATION AND OUTLINE OF THE THESIS}

\subsection{Motivation}

During the past decade, nanomedicine, which involves the use of nanoscale or nanostructured materials in medicine, has emerged as the main driving force for medical innovations ${ }^{1}$. Au nanoparticles (AuNPs), which have been used for clinic purpose since 1970s, are considered the most promising nanoparticles (NPs) for biomedical applications for the relatively easy synthesis and high controllability over the size and morphology ${ }^{2}$. However, some fundamental mechanisms of the interaction of the AuNPs of biological medium, particularly the adsorption of proteins on AuNP surface, remains unclear. In particular, the nanotoxicity of AuNPs, is a critical concern to the human health ${ }^{3}$. Despite the current intense experimental studies on the protein-AuNP interactions, some fundamental mechanisms remain unclear, which require nanoscale accuracy on both the length $(\mathrm{nm})$ and time (ns). Therefore, my research project applied molecular dynamics (MD) simulations to model and investigate the atomistic details of the protein-AuNP interactions, which can be used to complement and guide the active experimental studies in this filed.

\subsection{Research Objectives}

The objective of this research is to use atomistic simulations to investigate the interaction between AuNPs of various shapes and surface characteristics with proteins. The specific objectives of the project include:

(1) To validate the applicability of embedded-atom method (EAM) potential in modeling metals in biosystem. 
(2) To study the arrangement of self-assembled monolayers (SAMs) on both flat and spherical AuNP surfaces as a function of temperature, SAM chain length and Au surface characteristics.

(3) To study the interaction between the protein and SAMs covered Au surfaces, including the influence of $\mathrm{Au}$ shapes, temperatures, and functional groups of SAMs on the adsorption of the protein.

(4) To identify the adsorption sites and adsorption energy of the protein on the Au surfaces covered by SAMs terminated with different functional groups and to reveal the atomistic details of the conformation change of the bound protein on AuNPs.

(5) To compare the simulation results with experimental observations and to propose a model for the protein-AuNP conjugate formation.

\subsection{Thesis Contents and Structure}

This thesis is organized as follows: Chapter 1 is to introduce the research motivation, research objectives and the thesis outline. Chapter 2 is the literature review of previous research including nanomedicine, AuNPs, and AuNP-protien interaction studied by experiments and MD simulations. Chapter 3 is the introduction of the methodology used in this thesis research, including the basics of MD, the software, the force field and the model construction. Chapter 4 is the results and discussion. Chapter 5 is the conclusion and future work. 


\section{CHAPTER 2 LITERATURE REVIEW}

\subsection{Nanomedicine}

As defined by the National Institutes of Health of the USA, the application of nanotechnology for diagnosis, monitoring, treatment and control of biological systems has been referred to as "nanomedicine" 4 . The basic nanotechnology approaches for medical application date back to several decades ago. For example, Grefet al. developed the first long circulating stealth polymeric nanoparticle in $1994^{5}$; Bruchez et al. developed the first quantum dot bioconjugate in $1998^{6}$; and Cui et al. developed the first nanowire nano sensor which dates back to $2001^{7}$. In the future, nanomedicine will address many important medical problems by using nanoscale-structured materials and simple nano devices. Miniaturization of medical tools using nanomedicine will provide more accurate, more controllable, more versatile, more reliable, and more cost-effective approaches to enhance the quality of human life ${ }^{8}$.

The publication number on nanomedicine has risen from some ten articles per year in 1990 to more than 1,200 in the year 2004 as shown in Figure 2-1 ${ }^{1}$. European Patent Office also revealed that the patents on nanomedicine have skyrocketed from 220 in 1993 to 2,000 in 2003. Together with the research efforts, considerable commercialization efforts of nanomedicine are also taking place around the world. According to a global survey ${ }^{1}$, there were more than 150 start-ups and small and medium enterprises pursuing focused nanomedicine R\&D projects and 38 nanotechnology-enable products on the market in 2006 with total sales valued at $\$ 6.8$ billion ${ }^{1}$.

\section{The figure has been removed due to}




\section{copyright issues}

Figure 2-1. Nanomedicine publications and patents worldwide ${ }^{1}$.

Currently, the dominance of research on nanomedicine focuses on the use of NPs (particles of the size less than $1,000 \mathrm{~nm}$ ) in a broad range of biological applications, including drug delivery, drugs and therapy, in vivo imaging, in vitro diagnostic, active implants, etc ${ }^{9}$. This thesis will focus on the application of AuNPs in the biological environment, because AuNPs have been proved to be superior to other types of NPs for medical applications due to a few attractive properties. First, AuNPs can be fabricated with relatively low size dispersity. For example, AuNPs with a wide range of core sizes $(1-150 \mathrm{~nm})$ can be fabricated easily with controlled dispersity ${ }^{10}$. If AuNPs can be readily fabricated with sizes commensurate with biomolecules such as proteins and DNA, it will facilitate their integration into biological systems. Second, functional diversity can be readily achieved by the creation of multifunctional monolayers, allowing multiple functional moieties such as nucleic acids and targeting agents to be placed onto the particle surface ${ }^{11}$. Thirdly, AuNPs show enhanced permeation and retention in tumor tissue compared to normal tissue ${ }^{12}$. Moreover, the high surface area to volume ratio of AuNPs is advantageous for loading drugs molecules on the particle surface, leaving room to also attach recognition molecules such as antibodies, and thus enhance targeting to the desired location $^{13}$. Finally, the cytotoxicity, bio-distribution, and in vivo excretion properties can be modulated by regulating the particle size and surface functionality of AuNPs ${ }^{14}$. 


\subsection{AuNPs}

\subsubsection{Synthesis of AuNPs}

AuNPs can be prepared by both "top down" and "bottom up" approaches. For "top down" procedures, a bulk Au is systematically broken down to generate AuNPs of desired dimensions. In this case, particle assembly and formation is controlled by a pattern or matrix. The "top down" method is always limited concerning the control of the size and shape of particles as well as further functionalization ${ }^{15}$. In contrast, in the "bottom up" strategy, the formation of AuNPs originates from individual molecules which involves a chemical or biological reduction ${ }^{16}$. This chemical reduction method involves two steps: nucleation and successive growth. When the nucleation and successive growth are completed in the same process, it is called in situ synthesis; otherwise it is called seed-growth method ${ }^{17}$. In this section, the preparation of spherical or quasi-spherical AuNPs for the in situ synthesis method and the preparation of AuNPs having various sizes and shapes for the seed-growth method are reviewed respectively.

\section{(a) In Situ Synthesis}

In general, the preparation of AuNPs by chemical reduction contains two major steps: (i) reduction using agents such as borohydrides, hydrazine, hydroxylamine, saturated and unsaturated alcohols, (ii) stabilization by agents such as trisodium citrate dihydrate, sulfur ligands, phosphorus ligands, nitrogen-based ligands, oxygen-based ligands, dendrimers, and

polymers. The in situ synthesized AuNPs are also used for the seed-growth or further functionalization.

\section{- Turkevich method}


Citrate reduction of AuIII to Au0 in water was introduced by Turkevitch et al. in $1951^{18}$, which is a method that is still used nowadays. In this method, citrate can serve as both stabilizing and reducing agent or as a stabilizing agent only. In the first condition, using $\mathrm{HAuCl}_{4}$ solution, the AuNP with the size about $20 \mathrm{~nm}$ could be obtained. In 1973, Frens ${ }^{19}$ published an improvement and a broad size range of AuNPs (from 15 to $150 \mathrm{~nm}$ ) were obtained by controlling the trisodium citrate to Au ratio. Recently, Kimling et al. ${ }^{20}$ have improved the Turkevich-Frens method by noting that a high concentration of citrate more rapidly stabilizes AuNPs of smaller sizes, whereas a low concentration of citrate leads to large-size AuNPs and even to the aggregation of AuNPs.

Another situation is to apply citrate as a stabilizing agent only. In general, citrate plays a role as a stabilizing agent requiring relatively high temperatures due to its weak reducing strength. However, Natan's group introduced a method which can prepare AuNPs at room temperature. They used citrate as a stabilized agent only and $\mathrm{NaBH}_{4}$ as a reducing agent ${ }^{21}$. With this method, the size of AuNPs is tailored to $6 \mathrm{~nm}$, which compares with sizes beyond $20 \mathrm{~nm}$ using the traditional Turkevich method.

\section{- Brust-Schiffrin method}

Thiolate-stabilized AuNPs were first reported by Mulvaney and Giersig ${ }^{22}$, who showed the possibility of using alkanethiols of various chain lengths to stabilize AuNPs. The two-phase Brust-Schiffrin method, published in 1994, was the first method able to prepare the thiolates stabilized AuNPs via in situ synthesis ${ }^{23}$. Its high impact is due to (i) facile synthesis in ambient condition; (ii) relative high thermal and air stability of the AuNPs prepared in this way; (iii) repeated isolation and re-dissolution without aggregation or decomposition; (iv) control of the 
small size (less than $5 \mathrm{~nm}$ ) with narrow dispersity; and (v) relatively easily functionalization and modification by ligand substitution ${ }^{17}$.

Using Brust-Schiffrin method, the AuNPs are stabilized by relatively strong Au-S bonds, and their shapes are cuboctahedral and icosahedral. $\mathrm{HAuCl}_{4}$ and $\mathrm{NaBH}_{4}$ are usually used to yield thiolate-AuNPs. Functional thiolates can also be introduced using this method or achieved by the following equation ${ }^{24}$ :

$$
(\mathrm{RS}) \mathrm{nAuNP}+\mathrm{mR} \mathrm{R}^{\prime} \mathrm{SH} \rightarrow(\mathrm{RS}) \mathrm{n}-\mathrm{m}\left(\mathrm{R}^{\prime} \mathrm{S}\right) \mathrm{mAuNP}+\mathrm{mRSH}
$$

The reaction rate in AuNPs synthesis using Brust-Schiffrin method is much larger than that of the Turkevich AuNP synthesis. A direct consequence is that the size of the AuNPs synthesized using the Brust-Schiffrin method is much smaller than that of Turkevich method ${ }^{17}$.

\section{- Click reaction}

Using the famous "click” reaction is another useful protocol to synthesise AuNPs. In this method, a terminal alkyne and an azide are linked. "Click' reaction is also a way to form AuNPs with a precise number of metal atoms by using dendrimers which contain a pre-

organized number of internal ligands, leading to dendrimer-encapsulated AuNPs ${ }^{25}$. Super robust AuNPs were synthesized using PEG sorbitan fatty acid esters functionalized with lipoic acid, and could be stabled in the $\mathrm{pH}$ 1-14 range and under $\mathrm{NaCl}$ concentrations up to $5 \mathrm{M}^{3}$. These scaffolds show both strong coordination through the chelating thiols and van der Waals interactions.

\section{(b) Seed-Growth Method}

The seed growth usually involves two steps. In the first step, small-size AuNP seeds are prepared. In the second step, the seeds are added to a "growth" solution containing $\mathrm{HAuCl}_{4}$ and the stabilizing and reducing agents, then the newly reduced Au0 grows on the seed surface to 
form large-size AuNPs. The reducing agents used in the second step are always mild ones that reduce AuIII to Au0 only in the presence of $\mathrm{Au}$ seeds as catalysts, thus the newly reduced $\mathrm{Au0}$ can only assemble on the surface of the Au seeds, and no new particle nucleation occurs in solution $^{17}$.

The disadvantage of traditional in situ synthesis is that when the size increases it becomes out of control, and the shape is not controlled as well. Therefore, the seed-growth strategy has emerged as a very efficient method to synthesize monodispersed AuNPs with large sizes (up to $300 \mathrm{~nm}$ ) precisely and with well-defined shapes. For example, by using this method, Aslam et al. ${ }^{26}$ have produced the AuNPs with face centered cubic phase.

\subsubsection{Characterization of AuNPs}

\section{(a) Transmission Electron Microscopy (TEM)}

TEM is the most routine method to characterize the morphology, size, and shape of synthesized AuNPs. An example of AuNP image by TEM is shown in Figure 2-2. Those AuNPs are prepared from the seed-growth method ${ }^{27}$.

\section{The figure has been removed due to}

\section{copyright issues}

Figure 2-2. TEM image of larger gold particles prepared from seed: (a) $5.5 \pm 0.6$, (b) $8.0 \pm 0.8$, (c) $17 \pm 2.5$, and (d) $37 \pm 5 \mathrm{~nm}$ after separation of rods ${ }^{27}$.

\section{(b) Spectrophotometry}

Spectrophotometry is another important method used for AuNP characterization. With increase in particle size, the absorption peak shifts to longer wavelength and the width of 
absorption spectra is related to the size distribution range. Generally, because of surface plasmon resonance, spherical AuNPs display a single absorption peak in the visible range between $510-550 \mathrm{~nm}$, and show heavy absorption of visible light at $520 \mathrm{~nm}$. This gives brilliant red color to AuNPs, which varies according to their sizes ${ }^{28}$.

\section{(c) Optical Microscopy}

Optical microscopy is also used for AuNPs characterization. Large AuNPs (420 nm) can be imaged using an optical microscope in either phase contrast or differential interference contrast mode. Detection with an optical microscope only involves scattered light in dark-field microcopy. Small AuNPs only absorb light, provoking heating of the environment that can be

detected by photothermal imaging ${ }^{3}$. Those photothermal imaging can record local variations of the refractive index by differential interference contrast microscopy or by photoacoustic imaging using heat-induced liquid expansion.

\section{(d) Other Techniques}

Besides the most routine technique, several other imaging techniques are (i) fluorescence microcopy which allows detection at the single particle level, similar to the above mentioned plasmon based techniques, (ii) photothermal coherence tomography that is an optical analogue to ultrasound with relatively good penetration depth and resolution ${ }^{29}$, (iii) multiphoton surface plasmon resonance microscopy, which can illuminate the AuNPs by laser light in resonance with their plasmon frequency and generate an enhanced multiphoton signal measured in a laser scanning microscope ${ }^{30}$, (iv) X-ray scattering, which involves better contrast AuNP agents with high signal-to-noise ratio with X-ray computer tomograpy than organic molecules ${ }^{31}$, (v) gamma radiation using neutron activation ${ }^{3}$, and (vi) the UV-vis and IR (infrared) spectra, for an identification of the ligand IR spectroscopy. 


\subsubsection{Self-Assembled Monolayers (SAMs) on AuNPs}

The AuNPs are not simple hard spheres as what one might imagine. Typically, AuNPs are formed in the presence of ligand molecules that bind to the AuNPs and form the surface capping groups of the $\mathrm{NPs}^{32}$. Those ligands are called self-assembled monolayers (SAMs). By definition, SAM of organic molecules are molecular assemblies formed spontaneously on surfaces by adsorption from solution onto a solid substrate and are organized into more or less large ordered domains ${ }^{33}$. SAMs are created by the chemisorption of "head groups" onto a substrate followed by a slow organization of "tail groups" that assemble far from the substrate ${ }^{34}$.

The ligand molecules are essential to AuNPs for the following reasons. First, SAMs help stabilize AuNPs with a narrow size distribution and prevent their aggregation, because they can lower the surface free-energy of the substrate. Second, these surface stabilizing ligands confer to the AuNP solubility ${ }^{35}$. Furthermore, surface modification is an important method to enable AuNPs for various biomedical applications, especially for drug delivery ${ }^{9}$. Finally, SAMs can make AuNPs biocompatible in vivo.

Of these systems, alkanethiol SAMs formed on Au surfaces have been the most extensively studied. Since its discovery in the early 1980s, the properties of alkanethiol molecules chemisorbed on an $\mathrm{Au}$ (111) substrate have received enormous attention ${ }^{36}$. Considering the relative ease with which it can be prepared, the structural order it achieves is remarkable. The flexibility in controlling the functional groups of alkanethiol chains makes the alkanethiol-Au system so far the most versatile system yet devised in terms of surface property control and modification. The characterization of this system has been carried out using a variety of techniques, including $\mathrm{TEM}^{37}$, Fourier-Transformed Infrared Spectroscopy ${ }^{38}$, Helium Beam Diffraction $^{39}$, Macroscopic Wetting Experiments ${ }^{40}$, X-ray Diffraction ${ }^{41}$, etc. The consensus that 
has emerged from these studies is that the sulfur atoms coordinate strongly to the Au surface and, in the case of an ideal Au (111) substrate, form a slightly distorted $(\sqrt{ } 3 \mathrm{x} \sqrt{ } 3) \mathrm{R} 30^{\circ}$ triangular lattice ${ }^{42}$. The hydrocarbon tails self-organize into a well-ordered monolayer with chains tilted uniformly toward their next nearest neighbors. However, in spite of all these investigations, there still remain many unanswered questions. For instance, a quantitative understanding of the structural changes of SAMs occurring in the system at various temperatures is lacking, and the nature of the low temperature crystalline structures of SAMs has not been established yet. Most importantly, the influences of the structure and functionality of SAMs on protein adsorption on AuNPs remain to be explored.

\subsubsection{Nanotoxicity of AuNPs}

Despite the intense interests of NPs in diverse biomedical applications, the toxicity of nanomaterials, or the so-called "nanotoxicity", has become a rising issue. Although many studies have demonstrated adverse effects of nanomaterials in in-vitro cellular systems, at present, the biocompatibility and risks of exposure to nanomaterials is still not clearly understood and no general conclusion can be drawn ${ }^{42}$.

For example, Alkilany et al. ${ }^{43}$ summarized the toxic studies on AuNPs and concluded that AuNPs of sizes 4,12, and $18 \mathrm{~nm}$ in diameter and capped with different agents showed non-toxic

effect to human leukemia cell line. On contrary, Goodman et al. ${ }^{44}$ found that cationic AuNPs of $2 \mathrm{~nm}$ diameter induced toxic effect on COS-1 cells after $24 \mathrm{~h}$ incubation, while the same AuNPs with negative charge did not. Furthermore, it was concluded by Patra et al. ${ }^{45}$ that some citrate capped AuNPs were found toxic to a human lung carcinoma cells, but not to a human liver carcinoma cells. 
In study on the effect of AuNPs shape and surface functionalization onto epithelial cells, it was found that rod shaped AuNPs were less toxic than spherical ones. These results suggest that cell type-nanoparticle specific interactions play the roll in toxicity. Therefore, it has been suggested that a systematic toxicity study must be carried out for each specific case under precise conditions before using AuNPs in human for biomedical diagnosis or drug delivery ${ }^{3}$, such as the understanding of the molecular mechanism of the interaction between AuNPs and biological system, such as proteins, nuclei acid and lipids.

\subsection{AuNP-Protein Interactions}

Upon contact with biological environments, it has been generally accepted that AuNPs will immediately coated with biomolecules, most often proteins, forming the commonly referred "protein corona" around the AuNPs ${ }^{46}$. This adsorption of proteins onto AuNPs can greatly affect the structure and function of proteins, giving rise to either beneficial effects or to unpredictable and potentially undesirable effects ${ }^{47}$. The nature of the protein corona has been a subject of significant investigation. However, it is still difficult to determine specific rules that govern the adsorption and conformation of proteins on AuNPs surfaces. Recent reports have confirmed that the physicochemical properties of AuNPs such as size, shape and surface morphologies have considerable effects on the composition of the protein corona ${ }^{48}$. In this section, the latest findings regarding the composition and linking of the protein corona are presented. The Protein-AuNPs interactions are discussed with the focus on: (a) The influences of AuNP size, shape, surface on the protein adsorption and (b) the conformational changes of proteins upon binding to AuNPs. 


\subsubsection{Formation of Protein-AuNPs Corona}

Understanding the formation and persistence of the protein corona is a complex task but of great importance for the elucidation, interpretation, and assessment of the biological effects of AuNPs $^{49}$. Proteins can be adsorbed on the surface of AuNPs because this is the method for AuNPs to decrease the surface energy. It has been shown that the protein corona is composed of an inner layer of selected proteins with a lifetime of several hours in slow exchange with the environment which is usually called the hard corona, and an outer layer of weakly bound proteins which are characterized by a faster exchange rate with the free proteins which is usually called the soft corona, as shown in Figure 2-3 $3^{50}$. Hard corona means that proteins are bound to the surface durably and tightly. In contrast, soft corona indicates that the proteins are less tightly bound to the surface and dynamic. Researchers believed that the "soft" corona forms on short time scales from seconds to minutes, and evolves to a "hard" corona over incubation times of the order of hours ${ }^{13}$. Due to the long lifetime of the hard protein corona, it is now believed that it is the hard corona rather than the pristine NP surface interacts with cellular receptors and defines the fate of AuNPs in a biological environment. Therefore, identification of the adsorbed proteins and their lifetimes and conformations at the AuNP surface is considered a vital research question with deep implications for the design of safe nanomedicines.

\section{The figure has been removed due to}

\section{copyright issues}

Figure 2-3. Protein corona on NPs ${ }^{50}$. 


\subsubsection{Protein-NP Linking}

So how will proteins be adsorbed on the NP surfaces? During this processes, chemical or physical adsorptions such as coordination, hydrogen bonding, van der Waals forces, electrostatic, and hydrophobic interactions take part in the formation of protein corona. Generally, strategies to link a protein to the NP can take four main approaches (Figure 2-4): (a) electrostatic adsorption, (b) conjugation to the ligand on the NP surface, (c) conjugation to a small cofactor molecule that the protein can recognize and bind, and (d) direct conjugation to the NP surface.

\section{The figure has been removed due to}

\section{copyright issues}

Figure 2-4. NP-protein labeling strategies: (a) electrostatic attachment of protein, (b) covalent attachment to the NP ligand, (c) attachment of a protein cofactor on NP, and (d) direct linkage of amino acid on the NP core.

\section{(a) Electrostatic Adsorption}

Figure 2-4 (a) shows the simplest and most widely used linkage approach consisting of electrostatic adsorption, as it requires no chemical reaction. Appropriate conditions such as the $\mathrm{pH}$ or charge screening via controlling the ionic strength of the medium for the NP ligands and protein side chains to become attracted to each other must be determined ${ }^{51}$. However, the protein may interact with the NP in any of a number of orientations or have the NP positioned at any number of labeling sites by this non-specific linkage. Therefore, in order to let a specific face of the protein targeted for adsorption on the NP surface, modification of NP surface chemistry has been applied, e.g., by changing the charge on the end of the NP ligand and the ligand hydrophobicity ${ }^{52}$. 


\section{(b) Linking to the NP Ligand}

Covalently linking a protein to the NP ligand is another method for NP-protein conjugation as shown in Figure 2-4 (b). The proteins can be adsorbed on the surface of AuNPs if the NP has many ligands that can react with the protein. The stoichiometry can be influenced by varying the ratio of the reaction. A population of $\mathrm{NP}$-protein conjugates with various protein-to-NP ratios is usually produced. This approach has been greatly advanced by careful control over the surface chemistry of the NPs. For example, Hainfeld et al. have been able to isolate AuNPs with exactly one reactive group by high-performance liquid chromatography ${ }^{53}$, which limited the coverage of proteins on the NP surface. However, some research also showed that when proteins are covalently linked to the NP, both the structure and function of the protein can be affected, and typically several different protein orientations are observed. In addition, this covalent linkage can also affect the charge distribution of the ligands and the overall charge of the NPs ${ }^{54}$.

\section{(c) Linking Using Specific Affinity of Protein for a Cofactor}

Alternatively, as shown in Figure 2-4 (c), NP-protein conjugation can be achieved by using specific labeling strategies in bio-conjugation, among which biotin-streptavidin binding is a classical linkage. The biotin-streptavidin interaction is almost as strong as covalent binding. Because there are a considerable variety of linkers that can be functionalized with biotin, it is a versatile way to achieve a specific linkage. In addition, binding a NP to a specific protein by antibodies is another attractive route ${ }^{55}$. Antibodies are suitable for NP labeling since they can specifically bind to a target protein. The NP is first linked to the antibody, and the NP-antibody conjugate can then bind to a specific protein. Conjugation of NPs to antibodies generally adopts a global labeling strategy, such as targeting any of the primary amines distributed over the entire 
antibody, or reducing the disulfides in the hinge region to have free thiols that can react with a $\mathrm{NP}^{35}$

\section{(d) Direct Reaction with NP Surface Atoms}

Figure 2-4 (d) showed another popular method by direct reaction of a chemical group on the protein without the use of a linker ${ }^{56}$. Long linkers which are larger than the NP or protein can decrease circulation times in the blood or cause problems in cellular uptake. Therefore, direct linkage to the protein is much more desirable. For AuNPs, direct reaction can be achieved by the Au-thiol chemistry where a protein with a cysteine covalently bonds to an AuNP. The conjugation requires only incubation of the two species together as the $\mathrm{Au}-\mathrm{S}$ bond is strongly favored, even over a S-S bond. This results in a short, direct link from the protein side chain to the NP surface. However, this can be problematic as NP labeling can potentially break up dithiols of protein, which can compromise stability of protein and lead to denaturation. In the case of proteins that have free cysteines close to the protein surface that are not tied up as dithiols, this is a highly convenient way to conjugate a protein to an AuNP. Similarly, for sulfur containing NPs such as $\mathrm{ZnS} / \mathrm{CdSe}$, cysteine can directly form a disulfide bridge with surface $\mathrm{S}$ $\operatorname{atom}^{57}$

\subsubsection{Protein-AuNPs Interaction}

\section{(a) The Influences of AuNP Size, Shape, Surface on the Protein Adsorption}

\section{- Effect of NP size}

The curvature of the NP surface, which is dependent on the size of the NPs, can have a significant influence on the adsorption of biomolecules, which undergo different conformational changes from their native structure with respect to those observed for the protein adsorbed onto flat surfaces of the same material ${ }^{58}$. AuNPs of various sizes (i.e., 5, 10, 20, 30, 
60, 80, and $100 \mathrm{~nm}$ ) were incubated sequentially with common blood proteins, including albumin, fibrinogen, $\gamma$-globulin, histone, and insulin ${ }^{59}$. According to the results, the AuNPs had significant interaction with each of the proteins; the binding constant and the degree of cooperativity of protein-AuNP binding depended on the AuNP size. It has also been confirmed that the thickness of the protein corona gradually increased with increasing size of the AuNPs. Cedervall et al..$^{60}$ used AuNPs with various hydrophobicities and probed the protein adsorption on their surfaces. According to their results, there was a distinct difference in the degree of protein surface coverage of the AuNPs depending on their size, with a larger degree of protein coverage on the larger particles. By decreasing the AuNP size from 200 to 70nm, a curvatureinduced suppression of the protein adsorption was observed. Klein et al ${ }^{61}$ claimed that the curvature of smaller AuNPs may entirely suppress the adsorption of certain proteins, especially for larger or less conformationally flexible proteins; hence, the AuNP size is very important parameters in defining the composition of the formed protein corona.

\section{- Effect of NP shape}

The shape of NP has a great impact not only on its physiochemical characteristics but also on the way that proteins adsorb onto its surface and consequently on the way that cells interact with it. For instance, the shape of AuNPs has a great impact on their interactions with cell layers; more specifically, a peak in cell association for $50 \mathrm{~nm}$ spherical AuNPs will be decreased by changing the shape of the AuNPs to the rod geometry ${ }^{62}$. Also, coating Au nanocages (AuNC) with proteins is a popular method in order to improve their suspension stability. More interestingly, AuNC can directly bind to target protein and affect the protein activity, exhibiting the potential in disease treatment ${ }^{63}$.

\section{- Effect of particle/protein ratios}


The particle agglomeration behavior can be different for varying particle/protein ratios ${ }^{64}$. Thus, the particle surface might not be independent of the protein concentration, and the determination of the protein affinity might be hindered by this fact. It has also been observed that, for some NPs, the protein concentration to NP surface area ratio has quite dramatic effects on the nature of the adsorbed protein corona. The patterns of binding observed at in vitro protein concentrations (3-10\% proteins) were very different from those observed at in vivo (55-80\% proteins) concentrations for SAMs covered $\mathrm{NPs}^{65}$.

\section{- Effects of hydrophobicity/hydrophilicity}

A clear correlation between the affinities of proteins for the surface of NPs and the extent of structural changes has been found ${ }^{66}$. Due to their high affinities for hydrophobic surfaces, adsorbed proteins can have a less native structure than when adsorbed on hydrophilic surfaces, leading to severe protein denaturation ${ }^{67}$. In addition, NP surfaces may take up proteins depending on their isoelectric points in a rather narrow $\mathrm{pH}$ range ${ }^{68}$. It has also been observed that an increase in electrostatic interaction is generally accompanied by a reduction of the modification of the native structure ${ }^{69}$. According to literature reports, hydrophobic interactions

tend to dominate the energy balance in most cases tested to date ${ }^{70}$; however, the effect of electrostatic interactions cannot be ignored ${ }^{71}$. Cedervall et al. ${ }^{60}$ showed that the number of protein molecules bound increases with the particle hydrophobicity and that there was a clear difference in the protein affinities for polymeric particles of different hydrophobicities.

\section{- Effect of functional groups}

Yusuke et al. ${ }^{72}$ investigated biomolecules adhesion on the NPs covered with (SAMs) carrying different functional groups, including methyl $\left(\mathrm{CH}_{3}\right)$, hydroxyl $(\mathrm{OH})$, carboxylic acid $(\mathrm{COOH})$, and amine $\left(\mathrm{NH}_{2}\right)$. The correlating cell adhesion are examined and they concluded that, 
adhesion of human umbilical vein endothelial cells to the NPs covered with SAMs was greatly affected by surface functional groups: cells adhered well to $\mathrm{COOH}-$ and $\mathrm{NH}_{2}-\mathrm{SAMs}$, whereas poorly to $\mathrm{CH}_{3}-$ and $\mathrm{OH}-\mathrm{SAMs}$.

\section{(b) Conformational Changes of Proteins Upon Binding to NPs.}

Numerous experimental results ${ }^{56,73}$ showed that when the protein is adsorbed on the surface of AuNPs, the natural properties of both NPs and proteins may change, which has a high tendency to induce some physiological changes, including the configuration of bound proteins. For example, Nishima et al ${ }^{74}$ showed the conformation change of protein when it interacts with AuNPs, where the BSA protein undergoes a more flexible conformational state on the boundary surface of AuNPs after bioconjugation.

Another study by M-E Aubin-Tam ${ }^{35}$ investigated the interaction between proteins and AuNPs, and the results showed the structure and even the function change of the protein since structure and function are closely linked in proteins. Also, Hamad-Schifferli et al. ${ }^{35}$ found that when the enzyme is covalently linked to NPs, the cleavage of RNA is significantly slower. Sastry et al. ${ }^{75}$ found that when they conjugated $3.5 \mathrm{~nm}$ AuNPs with pepsin, there was a proteolytic activity comparable to that of the free enzyme when incubated with hemoglobin. Huang et al. ${ }^{76}$ studied the proteolytic activity of trypsin attached on $13 \mathrm{~nm}$ AuNPs and their results showed that the enzyme had a lower activity and a different specificity for cleavage. Finally, Zhang et al. ${ }^{77}$ studied the interaction between citrate-modified AuNPs and lysozyme, and the results showed that the interaction induced the aggregation of proteins in physiological conditions. Experimental methods such as TEM, and UV-visible spectroscopy were applied to characterize the processes and found that $\mathrm{S}-\mathrm{S}$ bonds were broken and $\mathrm{Au}-\mathrm{S}$ bonds were formed which induced the conformation change of lysozyme on the surface of AuNPs. Eventually 
protein unfolding, protein-AuNPs assemblies, and protein aggregations were observed by the researchers ${ }^{49}$.

\subsection{Simulation Studies on NP-Biosystem}

In recent years, the interaction of nanomaterials with biological systems has become one of the most intriguing areas of basic and applied research. Currently, experimental approaches can only obtain partial information on binding models of nano-bio complexes, although various advanced techniques, such as spectrophotometer, scanning electron microscopy, TEM, and nuclear magnetic resonance have been widely used ${ }^{46,78}$.

Since there is still an urgent need for a comprehensive study on the adsorption of proteins on AuNPs, it is expected that studies based on MD simulations could unveil the mystery of the atomistic details of the AuNP-protein interaction, specifically on the following concerns, all of which are difficult to be achieved using traditional experiment methods:

- It has been suggested that the surface energy of AuNPs can significantly influence the adsorption of proteins through experimental studies. However, a direct correlation between surface energy and protein adsorption cannot be established experimentally, which needs to be supported by MD simulations.

- It has been suggested that AuNPs with different shapes and surface characteristics will influence the protein adsorption, however, previous experiments still could not provide the fundamental atomistic mechanisms on this issue.

- The adsorption process, especially the specific sites of proteins and the binding energy of proteins on the AuNP surface are also hard to be studied by the experiments. Therefore, further MD studies are necessary to reveal the adsorption mechanism. 
In this regard, modeling and simulation efforts, particularly atomistic level modeling and simulation such as MD, have been widely used as important complementary to the experimental investigations. Quantum MD approaches can accurately describe the molecular system of NPs and proteins, but are not able to reach the relevant time and length scales needed for depicting large systems till equilibration ${ }^{79}$. In comparison, classical MD simulations can be used to study large molecular systems and reach long time scales by using a simplified force field. Therefore, among the different sampling methods available, classical MD is by far the most widely used technique to investigate the characteristics of nano-bio interactions.

\subsubsection{Past MD Studies on AuNP-Biosystem Interactions}

In this section, examples of MD simulation studies on NP-biosystem will be discussed, including the interaction of DNA with AuNPs, the interaction of proteins with SAMs, the interaction of lipid bilayers with AuNPs, and the interaction of proteins with Au Surface. The major research findings are highlighted in this section with the aim of demonstrating the versatility of MD in elucidating NP-biosystem interactions at the atomic level.

\section{(a) DNA-AuNP System}

DNA-functionalized Au surfaces and NPs have been used for a wide variety of applications including sequencing, drug discovery and bio-sensing ${ }^{80}$. Since the conformation of DNAs on surfaces and their interaction with neighboring DNAs significantly affect their properties, there have been many computational studies aimed at elucidating the DNA structures and

thermodynamics. Schatz et al. ${ }^{81}$ used MD simulations to study a $2 \mathrm{~nm}$ AuNP that was functionalized with four single stranded DNAs at the atomistic level. The DNA strands, which were attached to the faces of a 201-atom, truncated, octahedral gold particle using the six- 
carbon alkyl-thiolate linker, were found to be perpendicular to the surface of the particle, with the alkane chain lying on the surface, as shown in Figure 2-5. There were no significant hydrogen bonding interactions between the adsorbed DNAs during the simulation. The time averaged radius of gyration was also determined from the simulations which were found to be in good agreement with recent experimental results. It was also found that the sodium concentration within $30 \AA$ of the AuNP is about $20 \%$ higher than the bulk concentration. This increase in sodium concentration provided a clue to explain the increase of the melting temperature of DNA found in experiments for DNA-linked AuNPs aggregates.

\section{The figure has been removed due to}

\section{copyright issues}

Figure 2-5. Structure of DNA-AuNP and snapshots during simulation.

\section{(b) Protein-SAM System}

The adsorption of proteins to SAM surfaces is of great importance in the field of biomaterials because of its governing role in determining cellular responses to implanted materials and substrates for tissue engineering and regenerative medicine ${ }^{82}$.

Xie et al. ${ }^{83}$ applied all-atom MD simulations to investigate the neuromedin-B protein adsorption on the SAMs. The force-distance profiles show that the surface resistance to peptide adsorption is mainly generated by the water molecules tightly bound to surfaces via hydrogen bonds, but surfaces themselves may also set an energy barrier for the approaching protein. As shown in Fig. 2-6 (a), the peptide is repelled from the SBT-SAM surface and the SBT-SAM is more disordered than the other two kinds of SAMs. From Fig. 2-6 (b), it can be seen that the peptide is not adsorbed on the OH-SAM, but stays closer to the surface compared with the case 
for the SBT-SAM. Fig. 2-6 (c) shows that the peptide is adsorbed on the CH3-SAM with binding residues of His8, Trp4, Asn2, Phe9 and Ala5.

\section{The figure has been removed due to}

\section{copyright issues}

Figure 2-6. Final snapshot of the three peptide/SAMs system.

\section{(c) Lipid Bilayers-AuNP System}

In drug-delivery applications, AuNPs are required to enter biological cells in order to reach the targeted cellular compartments. Thus, during this process, AuNPs have to cross the cell membrane which includes a hydrophobic barrier. It has been demonstrated that the interaction of AuNPs with cell membranes tremendously influences their cellular uptake as well as their cytotoxicity $^{84}$. Therefore, a comprehensive investigation of the interaction of cell membranes with AuNPs at the atomic level is a crucial task in better understanding the effects of metallic nanoparticles in biological applications. Considering that the NPs first encounter the cell membrane on their way into the cell, the study of the NPs interacting with the membrane is an important topic for an understanding of the cytotoxicity. Liu et al. studied the effect of AuNPs surface charge on the membranes ${ }^{85}$, and the formation of holes on bilayer membranes caused by $\mathrm{AuNPs}^{86}$. In their work, only the electrostatic Coulomb forces due to the charged species were taken into account for the interaction between the AuNPs and the membranes. The AuNPs began to move down toward the bilayer very quickly, and when they were in contact, the AuNPs soon penetrated into the bilayer interior, as shown in Figure 2-7.

\section{The figure has been removed due to}




\section{copyright issues}

Figure 2-7. Snapshot of the interaction between the metal nanoparticles and the membrane.

\section{(d) Protein-Au Surface System}

Verde et al. ${ }^{87}$ applied all-atom MD simulations to investigate the adsorption of solvated protein approaching an Au surface, and the protein approaching an $\mathrm{Au}(001)$ surface in vacuum. Figure 2-8 shows the adsorption of proteins on Au surface in vacuum environment. In the study, they linked the behavior of proteins observed during the adsorption stage to their flexibility and stability in solution, and concluded that when the proteins are solvated, strong adsorption on Au takes place only if the proteins have high conformational flexibility and low conformational stability. In contrast, adsorption from vacuum occurs in all cases, despite low flexibility and high stability. This highlights the role of the solvent in adsorption, possibly via its mediation of protein/surface interactions.

\section{The figure has been removed due to}

\section{copyright issues}

Figure 2-8. Final adsorbed conformation of 6NGBP on Au (001) from MD simulations in vacuum at $310 \mathrm{~K}$. The tube represents the backbone of the protein. Adsorbed sections are shown in blue. The texture of the Au surface [in yellow] is omitted from the top views for clarity in the first figure ${ }^{87}$.

\section{(e) Protein-SAM-Au System}

In quite a number of recent studies, empirical all-atoms force field methods were successfully applied to determine the preferred orientations of surface bound proteins ${ }^{88}$. By using MD simulation to study the adsorption of the proteins IgG and cytochrome c (Cyt-c), Zhou et al. ${ }^{89}$ are able to show that there is a surface charge-driven mechanism of protein 
orientation on SAMs covered Au surface. The authors found the preferred orientation of Cyt-c with its dipole moment oriented orthogonally to the surface at high surface charge densities and a slightly tilted orientation at lower charge densities (Fig. 2-9).

\section{The figure has been removed due to}

\section{copyright issues}

Figure 2-9. Cyt-c configurations on Au surface covered with carboxyl-terminated SAMs. The SAMs with increasing dissociation degrees of the terminal carboxyl groups have been investigated, i.e. increasing surface charge densities: A) $5 \%$, B) $25 \%$

\subsubsection{Force Field (FF) for Protein-AuNP Simulation Studies}

\section{(a) FF for Biosystem Simulation}

FF is a crucially important parameter for the MD simulation and various FFs have been applied in different studies on biosystems. The applicability of several commonly used biomolecular FFs for the simulation of protein adsorption on hydrophobic and hydrophilic SAMs covered AuNPs has been systematically evaluated by Latour and colleagues ${ }^{90}$ for CHARMM19, and CHARMM22, Optimized Potential for Liquid Simulations (OPLS)-AA, and AMBER94 $\mathrm{FFs}^{91}$. In the latter study by Collier et al., ${ }^{91}$ the FF parameters and partial charges of the SAMs (-OH and $-\mathrm{COOH})$ were assigned by analogy to amino acids with similar functional groups. The authors compared experimental findings with their computed free energies of adsorption and qualitative behavior of peptides on different SAM surfaces such as the change in conformation upon adsorption and the orientation on the surface. This study demonstrated that although some FFs perform reasonably well, none of the FFs capture the specific interaction properties of the SAM-water interface. In particular, systematic overestimation of the binding strength of hydrophobic peptides and underestimation for 
negatively charged peptides was observed. On the other hand, it is also noted that altering FF parameters to reproduce the properties of adsorbed peptides unavoidably led to alteration of peptide behavior in solution. These results suggested that for accurate simulations of peptide adsorption a new FF strategy was required.

A new approach, the dual-FF, was proposed by Biswas et $\mathrm{al}^{92}$. In this FF, different sets of non-bonded parameters such as atomic partial charges and parameters of the Lennard-Jones potential, were used to represent interactions between proteins and SAMs in simulations. In addition, extended experimental benchmarks of binding free energies of peptides with different sequences on various functionalized SAM surface interfaces by Wei ${ }^{93}$ were used for optimization of the parameters for amino-acids.

\section{(b) Polarization Effect}

The metal polarization of AuNPs in the presence of the charges of a solute, has a significant effect on protein adsorption and, therefore, efforts have been put into integrating polarization effects into FF models. The simplest way to introduce the induced polarization of the metal surface in a computational model is using a classical image-charge approximation for a charge and a zero-potential metal surface in a continuum dielectric medium with an interaction energy expressed by Coulomb's law between the charge and its image of the opposite sign as shown in Figure 2-10. Heinz et al. ${ }^{94}$ exploited this scheme to estimate polarization effects a posteriori from non-polarizable fully atomistic MD simulation of a water-peptide-Au system. Although implementation of the image-charge approximation in an all-atom MD simulation is straightforward, it is impractical for large systems due to the increased computational load that scales with the number of interacting particles. In an alternative approach, which can be incorporated into any commonly used MD energy function, virtual dipoles or rods that can 
adjust their position in response to the external electrostatic field are introduced on all the surface atoms. Each virtual dipole is constrained at one end to a real surface atom, and depending on the model, can either change the magnitude of its dipole moment or its orientation (see Fig. 2-10 (b)), and hereby screen the external electrostatic field. A model with virtual rigid rods for simulation of the polarization of a metal surface was proposed and implemented by Iori \& Corni ${ }^{95}$ and used in the GolP family of FFs optimized for Au surfaces .

\section{(c) FF Used for Metal-Protein Interactions}

The first optimized sets of Lennard-Jones parameters to include metal-protein interactions were developed on the basis of DFT calculations by Vila Verde et al ${ }^{87}$. In the GolP FF, which is based on the OPLS FF, additional parameters describing the interactions of biomolecular groups with the Au surface are parameterized from experimental studies of adsorption energy. To reproduce the binding energy and orientation of small molecular fragments, the authors included and optimized a set of additional parameters that describe the van der Waals interactions with $\mathrm{Au}$ as well as stronger, chemical-like bonds between aromatic groups and $\mathrm{Au}$ atoms in the form of a Lennard-Jones function. Recently, Hoefling et al. ${ }^{96}$ applied the GolP FF in MD simulations of the adsorption of amino acids, as well as several proteins, on Au surface.

Furthermore, the ProMetCS ${ }^{97}$ continuum solvent model describes protein-surface interactions in atomic detail using the GolP Lennard-Jones interaction parameters together with an image charge model for protein-metal surface electrostatic interactions. Following the same strategy, a new force-field, GolP-CHARMM ${ }^{98}$ has recently been developed on the basis of the CHARMM FF for studying the interaction of proteins with $\mathrm{Au}$ (111) and Au (100) surfaces. In GolP-CHARMM, special attention has been paid to the correct description of the properties of the water molecules adsorbed on Au surface, with the properties being compared with ab initio 
MD simulations ${ }^{99}$. However, this force field is still infeasible for Au surfaces other than (111) and (100).

\section{(d) Embedded-Atom Method (EAM) for AuNPs}

Semi-empirical force fields based on embedded-atom method (EAM) for metals including Au have been well developed since $1980 \mathrm{~s}^{100}$ and implemented in general MD codes such as LAMMPS $^{101}$, which can accurately reproduce important properties of those metals such as surface energy. Therefore, the protein-AuNP system with MD simulation combining the best of two worlds is a very good choice, e.g., to use EAM potential for Au-Au interactions while maintaining the interactions among biomolecules with the force fields specifically designed for biomolecular systems such as proteins and lipids. It is worth mentioning that although the polarization influence is not fully considered in EAM potentials, the method to include polarization influence of the Au surface is mainly an independent analysis, i.e., with the image charge added to the Au surface manually ${ }^{95}$, can be integrated to EAM potentials whenever needed. By integrating EAM potential in modeling Au with biomolecules, the rich library of EAM potentials can also be used to model other metals in bio-systems, which is extremely useful when a more accurate, or specially developed potential for a specific purpose is not available. With this method, the SAMs and protein adsorption on different Au surfaces are able to be compared, e.g., Au (111), (110) and (100).

\section{The figure has been removed due to}

\section{copyright issues}

Figure 2-10. Models of polarizable Au surfaces ${ }^{95}$. 


\subsubsection{Challenges of Nano-Bio System Studies by MD Simulation}

Classical MD simulations are very helpful in understanding many molecular phenomena at atomic detail. However, the capabilities and limitations of the MD method, as of other sampling methods, must be known in order to set up a simulation and interpret the results obtained properly.

On one hand, researchers should pay attention to the simulation time required to reproduce an adsorption event on a molecular scale. Even though a simulation that captures the dynamics of a system for several nanoseconds is helpful for structural refinement of molecules or for understanding the initial stages of a process, many molecular events take place over much longer time scales. In one paper, Wei et al. ${ }^{102}$ stressed the necessity of very long simulations to be able to investigate a complete adsorption process on a surface in detail.

On the other hand, a bottleneck in the simulations of protein-surface systems is sampling. With classical MD simulations, complete sampling of the adsorption dynamics is not possible. Also, simulations of protein-surface interactions are still often not feasible due to the large size and complexity of proteins. Immersing the whole AuNP-protein system into water environment is challenging, considering how to handle the models with thousands of water molecules. Owing to those challenges, some simplifications were usually made in those previous MD models. For example, some previous simulation studies used united atom model for the $\mathrm{CH}_{2}$ and $\mathrm{CH}_{3}$ groups ${ }^{103}$, ignoring the effect of hydrogen atoms, while some other studies ignored $\mathrm{Au}-\mathrm{Au}$ interactions by fixing the sulfur head groups on the AuNP surface ${ }^{104}$. Also, other studies ignored the characteristics of AuNP, the surface of which should be covered by the SAMs. Considering all those limitations, further studies are necessary to develop simulation protocols, evaluate them and optimize suitable parameters. 


\section{CHAPTER 3 METHODOLOGY}

\subsection{Molecular Dynamics Simulation}

The principal tool used in this research is molecular dynamics (MD) simulation. This computational method calculates the time dependent behavior of a molecular system, and all the calculations are based on the Laws of Classical Mechanics. The equations of motion are solved numerically to follow the time evolution of the system, allowing the derivation of kinetic

and thermodynamic properties of interest by means of "computer experiments"105. MD simulation is very similar to real experiments, in which we measure a statistical property for a given system or predict the average behavior of a system when one or some of the system's statistical properties are known. Therefore, in MD simulation, the same steps as in experiment are followed:

1. To prepare the sample: In MD simulation, a system containing a particular number of atoms with particular volume, temperature, energy as needs to be created. Then this system needs to be initialized by assigning the initial positions and velocities to all the atoms in the simulation cell.

2. To conduct the experiment: In MD simulation, the experiment is performed by solving Newton's equations of motion for all the atoms. This step is critical and also the most time consuming.

3. To characterize the material properties: The force obtained from step 2 is used to update the new position and velocity of each atom. The response of the sample to external stimuli can thus be recorded and used to extract various properties.

And repeat step 2 and 3 until the desired length of time is reached. 


\subsubsection{The Advantages of Molecular Dynamics}

Computer simulations are carried out in the hope of understanding the properties of assemblies of molecules in terms of their structure and the microscopic interactions between them, which can serve as a complement to conventional experiments. The two main families of simulation technique are molecular dynamics (MD) and Monte Carlo (MC); additionally, there is also a whole range of hybrid techniques which combine features from both. In this study, MD simulation is concentrated, because the obvious advantage of MD over MC is that it gives a route to dynamical properties of the system: transport coefficients, time-dependent responses to perturbations, rheological properties and spectra.

Computer simulations act as a bridge (see Figure 3-1) between microscopic length and time scales and the macroscopic world of the laboratory: a guess is provided at the interactions between molecules, and "exact" predictions of bulk properties are obtained ${ }^{106}$. The predictions are "exact" in the sense that they can be made as accurate as one like, subject to the limitations imposed by our computer budget. At the same time, the hidden detail behind bulk measurements can be revealed. Simulations act as a bridge in another sense: between theory and experiment. The model and theory can be tested by comparing with experimental results. The simulations can also be carried out on the computer that are difficult or impossible in the laboratory, for example, working at extremes of temperature or pressure.

\section{The figure has been removed due to}

\section{copyright issues}

Figure 3-1, Simulations as a bridge between microscopic and macroscopic (left); (b) theory and experiment $(\text { right })^{106}$. 
Ultimately, direct comparisons can also be made with experimental results made on specific materials, in which case a good model of molecular interactions is essential. The aim of MD simulation is to reduce the amount of fitting and guesswork in this process to a minimum. On the other hand, if one simply wants to discriminate between good and bad theories, it is not necessary to have a perfectly realistic molecular model; MD models that contain the essential physics may be quite suitable.

\subsubsection{Molecular Dynamics Algorithm}

In MD simulations, the time evolution of a set of interacting particles is followed via the solution of Newton's equations of motion, where $\mathbf{r}_{i}(t)=\left(x_{i}(t), y_{i}(t) z_{i}(t)\right)$ is the position vector of $i$ th particle and $\mathbf{F}_{i}$ is the force acting upon $i$ th particle at time $t$ and $m_{i}$ is the mass of the particle.

$$
\mathbf{F}_{i}=m_{i} \frac{d^{2} \mathbf{r}_{i}(t)}{d t^{2}}
$$

'Particles' usually correspond to atoms, although they may represent any distinct entities (e.g. specific chemical groups) that can be conveniently described in terms of a certain interaction law. To integrate the above second order differential equations, the instantaneous forces acting on the particles and their initial positions and velocities need to be specified. Due to the manybody nature of the problem, the equations of motion are discretized and solved numerically. The MD trajectories are defined by both position and velocity vectors and they describe the time evolution of the system in phase space. For a system of $\mathrm{N}$ particles with coordinates $\mathrm{X}$ and 
velocities $\mathrm{V}$, the following pair of first order differential equations may be written in Newton's notation as:

$$
\begin{aligned}
& \mathrm{F}(\mathrm{X})=-\nabla \mathrm{U}(\mathrm{X})=\mathrm{MV}(\dot{t}) \\
& \mathrm{V}(\mathrm{t})=\mathrm{X}(\dot{t})
\end{aligned}
$$

The potential energy function $\mathrm{U}(\mathrm{X})$ of the system is a function of the particle coordinates $\mathrm{X}$. It is referred to simply as the "potential" in physics, or the "force field" in chemistry. The first equation comes from Newton's laws; the force F acting on each particle in the system can be calculated as the negative gradient of $\mathrm{U}(\mathrm{X})$. For every time step, each particle's position $\mathrm{X}$ and velocity V may be integrated with a method such as Verlet. Given the initial positions (e.g. from theoretical knowledge) and velocities (e.g. randomized Gaussian), we can calculate all future (or past) positions and velocities. Therefore, the aim of the numerical integration of Newton's equations of motion is to find an expression that defines positions $\mathbf{r}_{i}(t+\Delta t)$ at time $t+\Delta t$ in terms of the already known positions at time $t$. Because of its simplicity and stability, the Verlet algorithm is commonly used in MD simulations. The basic formula for this algorithm can be derived from the Taylor expansions for the positions $\mathbf{r}_{i}(t)$; it reads as in equation (4).

$$
\mathbf{r}_{i}(t+\Delta t) \cong 2 \mathbf{r}_{i}(t)-\mathbf{r}_{i}(t-\Delta t)+\frac{\mathbf{F}_{i}(t)}{m_{i}} \Delta t^{2}
$$

Equation (4) is accurate up to terms of the fourth power in $\Delta t$. Velocities can be calculated from the positions or propagated explicitly as in alternative leapfrog or velocity Verlet schemes. And the velocities can be used to determine the kinetic energy and temperature in the system ${ }^{107}$. As the particles move, their trajectories may be displayed and analysed, providing averaged properties. The dynamic events that may influence the functional properties of the system can be directly traced at the atomic level, making MD especially valuable in molecular biology. 
The exact trajectories correspond to the limit of an infinitesimally small integration step. It is, however, desirable to use larger time steps to sample longer trajectories. In practice $\Delta t$ is determined by fast motions in the system. Bonds involving light atoms (e.g. the $\mathrm{O}-\mathrm{H}$ bond) vibrate with periods of several femtoseconds, implying that $\Delta t$ should be on a subfemtosecond scale to ensure stability of the integration. Although the fastest and not crucial vibrations can be eliminated by imposing constraints on the bond length in the integration algorithm, a time step of more than 5 fs can rarely be achieved in simulations of biomolecules.

\subsubsection{Periodic Boundary Conditions}

Schematic representation of the Periodic Boundary Conditions (PBC) is shown in Figure 3$2^{108}$. Ideally, we would like to simulate molecular systems with a number of particles in the order of magnitude of the Avogadro number ( $10^{23}$ atoms). In this way, a small fraction of the atoms would be close to the boundary and we could be sure that the measured properties correspond to the interior (i.e., "bulk") atoms of our simulation cell. The largest MD systems that we can simulate today $\left(\sim 10^{6}\right.$ atoms $)$ are still far from this objective, but if the system does not have important electrostatic interactions (e.g., a Lennard-Jones system), good results can be obtained even using a small simulation cell. When electric charges are involved, it would be suitable to have a simulation cell at least as big as the Bjerrum length, the distance at which the electrostatic interactions between two elementary charges is comparable to the thermal energy. However, even if this requirement is met, we cannot always discard the influence of highly charged groups at greater distances. Additionally, at large distances, direct electrostatic calculations of every single pair of interaction become unpractical; the implementation of $\mathrm{PBC}$ 
alleviates this difficulty ${ }^{109}$. The introduction of PBC is equivalent to consider an infinite array of identical copies of the simulation box.

\section{The figure has been removed due to}

\section{copyright issues}

Figure 3-2: Schematic representation of the Periodic Boundary Conditions (PBC) ${ }^{109}$

Maintenance of the minimum-image convention also generally requires that a spherical cutoff radius for nonbonded forces be at most half the length of one side of a cubic box. The error of calculating short range non-bonded interactions for a particle within a cutoff RC can be made infinitely small by increasing this cutoff to infinity. However, for practical reasons, this is never done, a normal assumption for a good cutoff is that for any $r, r>\mathrm{RC}$, the intermolecular interaction should be zero. If this condition is not fulfilled, then a systematic error is introduced when calculating the potential energy. A variety of shifting or switching functions have been proposed that modify the Coulomb potential by smoothly truncation; however, even when some of these approaches lead to stable dynamics ${ }^{110}$, the relationship of these modified potentials to basic electrostatics is not clear. A better treatment of long range electrostatics taking into account PBC is therefore needed.

\subsubsection{Thermodynamic ensembles}

In statistical mechanics, a canonical ensemble is the statistical ensemble that represents the possible states of a mechanical system in thermal equilibrium with a heat bath at some fixed temperature $^{111}$. The system can exchange energy with the heat bath, so that the states of the 
system will differ in total energy. The following ensembles are commonly used in MD simulations:

1. Canonical ensemble (NVT): The principal thermodynamic variable of the canonical ensemble, determining the probability distribution of states, is the absolute temperature (T). The ensemble typically also depends on mechanical variables such as the number of particles in the system (N) and the system's volume (V), each of which influence the nature of the system's internal states. An ensemble with these three parameters is sometimes called the NVT ensemble. Here, the temperature has an average (macroscopic) value, while the total energy of the system is allowed to fluctuate.

2. Isothermal-isobaric (Gibbs) ensemble (NPT): NPT ensemble is a statistical mechanical ensemble that maintains constant temperature $\mathrm{T}$, and constant pressure $\mathrm{P}$, where the number of particles $\mathrm{N}$, is also kept as a constant. Here the pressure has an average value while the instantaneous volume of the system is allowed to fluctuate.

3. Microcanonical ensemble (NVE): In the microcanonical, or NVE ensemble, the system is isolated from changes in moles $(\mathrm{N})$, volume $(\mathrm{V})$ and energy $(\mathrm{E})$. It corresponds to an adiabatic process with no heat exchange. A microcanonical molecular dynamics trajectory may be seen as an exchange of potential and kinetic energy, with total energy being conserved.

However, the meaning of temperature in MD is a source of confusion. Commonly we have experience with macroscopic temperatures, which involve a huge number of particles. But temperature is a statistical quantity. If there is a large enough number of atoms, statistical temperature can be estimated from the instantaneous temperature, which is found by equating the kinetic energy of the system to $\mathrm{nkBT} / 2$, where $\mathrm{n}$ is the number of degrees of freedom of the 
system. A temperature-related phenomenon arises due to the small number of atoms that are used in MD simulations. For example, consider simulating the growth of a copper film starting with a substrate containing 500 atoms and a deposition energy of $100 \mathrm{eV}$. In the real world, the $100 \mathrm{eV}$ from the deposited atom would rapidly be transported through and shared among a large number of atoms $10^{10}$ or more with no big change in temperature. When there are only 500 atoms, however, the substrate is almost immediately vaporized by the deposition. Something similar happens in biophysical simulations. The temperature of the system in NVE is naturally raised when macromolecules such as proteins undergo exothermic conformational changes and binding. For comparison with experiments, NVT and NPT are more useful.

\subsection{Software}

\subsubsection{Materials Studio}

Materials Studio ${ }^{112}$ was used to construct the all-atom model of SAMs in this research. It is the software for simulating and modeling materials, which is developed and distributed by Accelrys Company. The interface of Materials Studio is shown in Figure 3-3:

\section{The figure has been removed due to}

\section{copyright issues}

Figure 3-3. Materials Studio software Interface

\subsubsection{VMD}

$\mathrm{VMD}^{113}$, represents Visual Molecular Dynamics, is a molecular visualization program for displaying, animating, and analyzing large systems using 3-D graphics and built-in scripting. It 
is also designed particularly for modeling, visualization, and analysis of biological systems such as proteins, nucleic acids, lipid bilayer assemblies, etc. It may be used to view more general molecules, as VMD can read standard Protein Data Bank (PDB) files and display the contained structure. VMD provides a wide variety of methods for rendering and coloring a molecule: simple points and lines, CPK spheres and cylinders, licorice bonds, backbone tubes and ribbons, cartoon drawings, and others. VMD can also be used to animate and analyze the trajectory of a molecular dynamics (MD) simulation. In particular, VMD can act as a graphical front end for an external MD program by displaying and animating a molecule undergoing simulation on a remote computer. In my research, the constructed models from Materials Studio of the protein molecule and the alkanethiol chains were converted to the LAMMPS data file format by VMD. The interface of VMD is shown in Figure 3-4:

\section{The figure has been removed due to}

\section{copyright issues}

Figure 3-4. VMD software Interface

\subsubsection{LAMMPS}

In my research, all the simulations were performed by using LAMMPS ${ }^{101}$ which met our requirement for the force field. LAMMPS, which is represent for "Large-scale Atomic/Molecular Massively Parallel Simulator", is a molecular dynamics program from Sandia National Laboratories which can work together with Python. For computational efficiency, LAMMPS uses neighbor lists to keep track of nearby particles. The lists are optimized for systems with particles that are repulsive at short distances, so that the local density 
of particles never becomes too large. On parallel computers, LAMMPS uses spatialdecomposition techniques to partition the simulation domain into small $3 \mathrm{~d}$ sub-domains, one of which is assigned to each processor. Processors communicate and store "ghost" atom information for atoms that border their sub-domain. LAMMPS is most efficient (in a parallel computing sense) for systems whose particles fill a 3D rectangular box with approximately uniform density.

\subsubsection{AtomEye}

AtomEye $^{114}$ was used for the visualization and image generation of all atomistic configurations in my study. It is a free atomistic visualization software for all major UNIX platforms. AtomEye can handle more than one million atoms on a PC with 1 GB memory. It is a robust, low-cost tool for surveying nanostructures and following their evolutions. In my study, all the result images are generated from AtomEye.

\subsection{Force Field}

\subsubsection{General Concept}

A force field, refers to the energy functional form and parameter sets used to calculate the potential energy of a system of atoms or coarse-grained particles in molecular mechanics and MD simulations. The parameters of the energy functions can be derived from experimental work and quantum mechanical calculations. "All-atom" force fields provide parameters for every type of atom in a system, including hydrogen, while "united-atom" interatomic potentials treat the hydrogen and carbon atoms in each terminal methyl and each methylene bridge as a single interaction center. "Coarse-grained" potentials, which are frequently used in long-time 
simulations of macromolecules such as proteins, nucleic acids, and multi-component complexes, provide even more crude representations for increased computational efficiency.

Atomic force field models and the classical MD are based on empirical potentials with a specific functional form, resembling physics and chemistry of studied systems. A typical force field, used in the simulations takes the following form ${ }^{115}$ :

$$
\begin{aligned}
U\left(\mathbf{r}_{1}, \cdots, \mathbf{r}_{N}\right)= & \sum_{\text {bonds }} \frac{a_{i}}{2}\left(l_{i}-l_{i 0}\right)^{2}+\sum_{\text {angles }} \frac{b_{i}}{2}\left(\theta_{i}-\theta_{i 0}\right)^{2}+\sum_{\text {torsions }} \frac{c_{i}}{2}\left(1+\cos \left(n \omega_{i}-\gamma_{i}\right)\right) \\
& +\sum_{\text {atom pairs }} 4 \varepsilon_{i j}\left[\left(\frac{\sigma_{i j}}{r_{i j}}\right)^{12}-\left(\frac{\sigma_{i j}}{r_{i j}}\right)^{6}\right]+\sum_{\text {atom pairs }} k \frac{q_{i} q_{j}}{r_{i j}}
\end{aligned}
$$

where in the first three terms summation indices run over all the bonds, angles and torsion angles defined by the covalent structure of the system, whereas in the last two terms summation indices run over all the pairs of atoms (or sites occupied by point charges), separated by distances and not bonded chemically. Physically, the first two terms describe energies of deformations of the bond lengths $l_{i}$ and bond angles $\theta_{i}$ from their respective equilibrium values $l_{i 0}$ and $\theta_{i 0}$. The third term describes rotations around chemical bond. The fourth term describes the van der Waals repulsive and attractive (dispersion) interatomic forces in the form of the Lennard-Jones 12-6 potential and the last term is the Coulomb electrostatic potential. Some effects due to specific environments can be accounted for by properly adjusted partial charges $q_{i}$ (and an effective value of the constant k) as well as the van der Waals parameters $\varepsilon_{i j}$ and $\sigma_{i j}$.

In this study, the total conformational energy ( $\left.\mathrm{V}_{\text {total }}\right)$ of the alkanethiol chain is given by:

$$
\mathrm{V}_{\text {total }}=\mathrm{V}_{\text {intra }}+\mathrm{V}_{\text {inter }}
$$


Here, $\mathrm{V}_{\text {intra }}$ represents the bonded interactions that arise from bond stretching $\left(\mathrm{V}_{\text {bond }}\right)$, bond bending $\left(\mathrm{V}_{\text {angle }}\right)$, and torsion $\left(\mathrm{V}_{\text {dihedral }}\right)$ :

$$
\mathrm{V}_{\text {intra }}=\mathrm{V}_{\text {bond }}+\mathrm{V}_{\text {angle }}+\mathrm{V}_{\text {dihedral }}
$$

The parameters for bonded interactions in my study are listed in Table 1, all the parameters were taken from the literature.

Table 1: Force Field Parameters ${ }^{116}$

\begin{tabular}{lll}
\hline Bond & $\mathrm{r}_{0}(\AA)$ & $\mathrm{K}\left(\mathrm{Kcal} / \mathrm{mol} / \AA^{2}\right)$ \\
\hline C-C & 1.530 & 186.188 \\
C-H & 1.111 & 258.571 \\
C-S & 1.816 & 222 \\
\hline & & \\
\hline Angle & Theta (degree) & $\mathrm{K}\left(\mathrm{Kcal} / \mathrm{mol}^{2} / \Theta^{2}\right)$ \\
\hline C-C-C & 111.0 & 446.433 \\
C-C-H & 110.1 & 288.696 \\
C-C-S & 114.5 & 485.344 \\
H-C-H & 108.4 & 297.064 \\
H-C-S & 111.3 & 385.764 \\
\hline Dihedral & $\varphi(\mathrm{rad})$ & $\mathrm{K}\left(\mathrm{Kcal} / \mathrm{mol}^{\left.-\mathrm{rad}^{2}\right)}\right.$ \\
\hline C-C-C-C & 180 & 0.4184 \\
C-C-C-H & 0 & 0.79416 \\
C-C-C-S & 180 & 1.00416 \\
H-C-C-H & 0 & 0.8368 \\
H-C-C-S & 0 & 0.8368 \\
\hline
\end{tabular}

\subsubsection{Lennard-Jones Potential}

From the equation above, $\mathrm{V}_{\text {inter }}$ is the non-bonded interaction modeled by 6-12 LennardJones potential. The Lennard-Jones potential (also referred to as the L-J potential, 6-12 potential, or 12-6 potential) is a mathematically simple model that approximates the interaction between 
a pair of neutral atoms or molecules. A form of this interatomic potential was first proposed in 1924 by John Lennard-Jones ${ }^{117}$. The most common expressions of the Lennard-Jones potential are:

$$
V=\sum_{\text {atom pairs }} 4 \varepsilon_{i j}\left[\left(\frac{\sigma_{i j}}{r_{i j}}\right)^{12}-\left(\frac{\sigma_{i j}}{r_{i j}}\right)^{6}\right]
$$

where $\varepsilon_{i j}$ is the depth of the potential well, $\sigma_{i j}$ is the finite distance at which the inter-particle potential is zero, $r_{i j}$ is the distance between the particles. $r_{i j}$ is the distance at which the potential reaches its minimum. At $r_{i j}$, the potential function has the value $-\varepsilon_{i j}$. The distances are related as $r_{i j} \approx 1.122 \sigma_{i j}^{117}$. These parameters can be fitted to reproduce experimental data or accurate quantum chemistry calculations. Due to its computational simplicity, the Lennard-Jones potential is used extensively in computer simulations. A graph of strength versus distance for the 12-6 Lennard-Jones potential is shown in Figure 3-5 ${ }^{117}$.

\section{The figure has been removed due to}

\section{copyright issues}

Figure 3-5. The strength versus distance for the 12-6 Lennard-Jones potential

The force field considering the water solvent was described by compute $\mathrm{lj} /$ charmm/coul/charmm/implicit in LAMMPS. It includes an additional $1 / \mathrm{r}$ term in the Coulombic formula to account for additional screening from the implicit water solvent for the simulation of biomolecules. The implicit solvation could significantly reduce the computational expense that arises from calculating the interactions between the explicit water molecules. The 
parameters of nonbonded Lennard-Jones potential interaction are listed in Table 2, and all the parametes were taken from the literature.

Table 2: Force Field Parameters of Lennard-Jones Nonbonded Interaction ${ }^{118}$

\begin{tabular}{lll}
\hline Atom & $\alpha(\AA)$ & $\mathrm{K}\left(\mathrm{Kcal} / \mathrm{mol} / \Theta^{2}\right)$ \\
\hline $\mathrm{C}$ & 3.5 & 0.066 \\
$\mathrm{H}$ & 2.5 & 0.03 \\
$\mathrm{~S}$ & 3.55 & 0.25 \\
\hline
\end{tabular}

\subsubsection{Morse potential}

The interaction between Au-S was modeled using Morse potential. The Morse potential ${ }^{119}$, named after physicist Philip M. Morse, is a convenient interatomic interaction model for the potential energy of a diatomic molecule. It is a better approximation for the vibrational structure of the molecule than the QHO (quantum harmonic oscillator) because it explicitly includes the effects of bond breaking, such as the existence of unbound states. The Morse potential can also be used to model other interactions such as the interaction between an atom and a surface. Due to its simplicity (only three fitting parameters), it is not used in modern spectroscopy. However, its mathematical form inspired the MLR (Morse/Long-range) potential, which is the most popular potential energy function used for fitting spectroscopic data. The Morse potential energy function is of the form:

$$
V(r)=D_{e}\left(1-e^{-a\left(r-r_{e}\right)}\right)^{2}
$$

Here $r$ is the distance between the atoms, $r_{e}$ is the equilibrium bond distance, $D_{e}$ is the well depth (defined relative to the dissociated atoms), and controls the "width" of the potential. The dissociation energy of the bond can be calculated by subtracting the zero point energy from the depth of the well. The force constant of the bond can be found by Taylor expansion of $V(r)$ 
around $r=r_{e}$ to the second derivative of the potential energy function, from which it can be shown that the parameter, $a$, is

$$
a=\sqrt{k_{e} / 2 D_{e}}
$$

where $k_{e}$ is the force constant at the minimum of the well. Figure 3-6 shows the Morse potential (blue) and harmonic oscillator potential (green) ${ }^{119}$.

\section{The figure has been removed due to}

\section{copyright issues}

Figure 3-6. The Morse potential (blue) and harmonic oscillator potential (green)

From the Figure 3-6, we can see unlike the energy levels of the harmonic oscillator potential, the Morse potential level spacing decreases as the energy approaches the dissociation energy. The parameters for Morse potential in my research are listed in Table 3, all the parameters were taken from the literature.

Table 3: Au-S Interaction (Morse Potential) ${ }^{103 a, 120}$

\begin{tabular}{llll}
\hline Atoms & De $(\mathrm{Kcal} / \mathrm{mol})$ & $\mathrm{r}_{\mathrm{e}}(\AA)$ & $\alpha\left(\AA^{-1}\right)$ \\
\hline $\mathrm{Au}-\mathrm{S}$ & 9.108 & 2.903 & 1.47 \\
\hline
\end{tabular}

\subsubsection{Embedded-Atom Method Potential}

For the Au-Au interactions, the Embedded-Atom Method (EAM) potential was used. The EAM potential of Au can accurately reproduce important properties of Au including the surface energy, which is of particular importance for this study. The EAM potential is an approximation describing the energy between atoms as a function of a sum of functions of the separation 
between an atom and its neighbors. In a simulation, the potential energy of an atom, i, is given by $^{100}$

$$
\mathrm{E}_{i}=\mathrm{F}_{\alpha}\left(\sum_{i \neq j} \rho_{\beta}\left(r_{i j}\right)\right)+\frac{1}{2} \sum_{i \neq j} \varphi_{\alpha \beta}\left(r_{i j}\right)
$$

where $r_{i j}$ is the distance between atoms $\mathrm{i}$ and $\mathrm{j}, \varphi_{\alpha \beta}$ is a pair-wise potential function, $\rho_{\beta}$ is the contribution to the electron charge density from atom $j$ of type $\beta$ at the location of atom $i$, and $F$ is an embedding function that represents the energy required to place atom $i$ of type $\alpha$ into the electron cloud. The EAM potential for Au using an improved force matching methodology shows good overall agreement to a range of properties including experimental lattice cohesive energy, lattice constant versus temperature, elastic constants, stacking fault energy, radial distribution function, and fcc/hcp/bcc lattice energy differences and a somewhat better agreement to surface energies over more traditional potential. Figure 3-7 and 3-8 ${ }^{100}$ show the pair potential, electron-density function, and embedding energy function of Au EAM potential.

\section{The figure has been removed due to}

\section{copyright issues}

Figure 3-7. The pair potential and electron-density function ${ }^{100}$.

\section{The figure has been removed due to}

\section{copyright issues}

Figure 3-8. The embedding energy function ${ }^{100}$. 


\subsection{Model Construction and Simulation Settings}

\subsubsection{Model Construction}

The all-atom model of alkanethiol chains with ten carbons as the chain backbone were constructed by Materials Studio. Identical to the experimental studies ${ }^{121}$, the alkanethiol chains with both methyl and carboxyl terminated functional groups were constructed. The Lysozyme (Lyz) protein molecule structure was retrieved from the PDB. Since all the simulations were performed by using LAMMPS in this study, the as constructed models of the protein molecule and the alkanethiol chains were converted to the LAMMPS data file format by VMD. The flat $\left(\begin{array}{lll}1 & 1 & 1\end{array}\right),\left(\begin{array}{lll}1 & 1 & 0\end{array}\right)$ and $\left(\begin{array}{lll}1 & 0 & 0\end{array}\right)$ Au surfaces as well as the spherical AuNP were created from LAMMPS directly. The SAMs were constructed by arranging individual alkanethiol chains on the Au surface, e.g., with an interval distance of $4.32 \AA$ in the $\mathrm{x}$ direction and $4.988 \AA$ in the $\mathrm{y}$ direction on (111) Au surface. The same method was used to construct the SAMs on the (110) and (100) surface as well, but the interval distance between single chains was $4.05 \AA$ in the $\mathrm{x}$ direction and $5.32 \AA$ in the y direction on (110) Au surface, and $3.7 \AA$ in the $\mathrm{x}$ direction and 5.7 $\AA$ in the y direction on Au (100) surface respectively.

The method to construct the carboxyl-terminated SAMs covered spherical AuNPs were shown in Figure 3-9. The AuNP with diameter of $4 \mathrm{~nm}$ was constructed in LAMMPS directly. The initial configuration was set up with two arrays of 14 x 16 carboxyl-terminated SAMs on both sides of the AuNP with sulfur atoms pointing towards the AuNP surface as shown in Figure 3-9 (a). The model was first relaxed at zero pressure for $100 \mathrm{ps}$ at $300 \mathrm{~K}$, but the SAMs could not cover the spherical AuNP surface evenly as shown in Figure 3-9 (b). In order to remove the extra SAMs and homogenize the distribution of SAMs on the AuNP, the temperature was 
increased to $1000 \mathrm{~K}$ and the model was relaxed for additional $180 \mathrm{ps}$. Only the SAMs that attached to the AuNP by the Au-S strong bonding remained on the AuNP surface and distributed evenly, while the extra SAMs have moved away from the AuNP surface. In this way carboxylterminated SAMs-AuNP was constructed as shown in Figure 3-9 (c), which was used as the initial model for simulating AuNP-AuNP and AuNP-protein interactions.

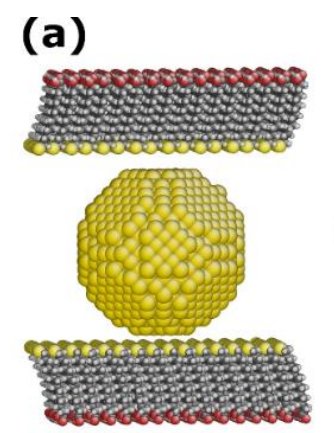

(b)

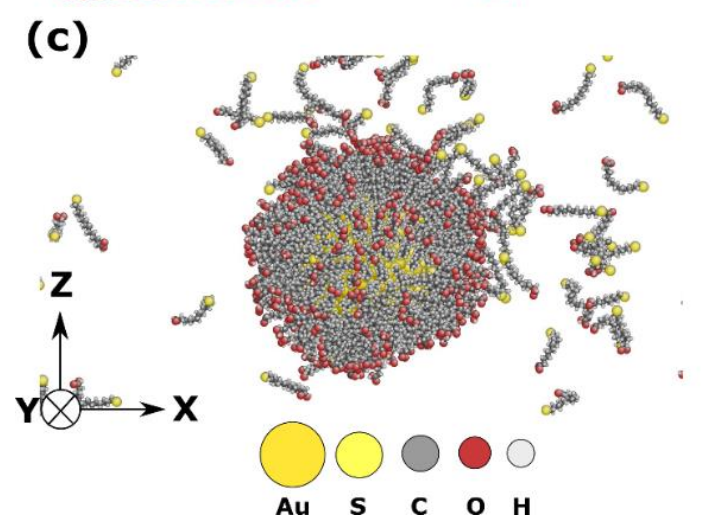

Figure 3-9. Atomistic configurations of an assembly of a spherical AuNP with two carboxyl-terminated SAMs. (a) The initial configuration; (b) the configuration after relaxation at $300 \mathrm{~K}$ for $100 \mathrm{ps}$; (c) the configuration after relaxation of additional $180 \mathrm{ps}$ at $1000 \mathrm{~K}$.

\subsubsection{System Geometry and Simulation Settings}

Periodic boundary conditions were applied to $\mathrm{x}$ and $\mathrm{y}$ directions while the $\mathrm{z}$ direction was kept free in all models. All simulations were performed in the canonical ensemble (constant number of ligands $\mathrm{N}$, volume $\mathrm{V}$, and temperature $\mathrm{T}$ ) at both $300 \mathrm{~K}$ and $600 \mathrm{~K}$. A time step of 1.0 
fs was used for integrating the equations of motion, which yielded good conservation of energy. The inner cutoff is $8.0 \AA$ while the outer cutoff is $10.0 \AA$.

For the model of a single methyl terminated alkanethiol chain on the Au (111) surface, the sulfur atom was initially positioned $4.72 \AA$ away from the Au surface. For the model of the protein adsorption on SAMs covered flat Au surface, the initial simulation cell was $\sim 170 \AA$ $\times 172 \AA$ in the in $\mathrm{x}$ and $\mathrm{y}$ directions for methyl-terminated SAMs, and $\sim 141 \AA \times 137 \AA$ in the $\mathrm{x}$ and y directions for carboxyl terminated SAMs and the SAMs were placed $5 \AA$ away from the $\mathrm{Au}$ (111) surface. All the simulations have been run for at least three times by varying the initial conditions such as initial position of the protein, e.g., the Lyz protein molecule have been positioned $24 \AA, 10 \AA$ and $5 \AA$ away from the SAMs surface respectively. While studying the interaction between carboxyl terminated SAMs cover Au surface, the protein has also been rotated by $180^{\circ}$ to study adsorption process of protein with different initial orientations. To study the interaction between the protein and two spherical AuNPs, the distance between the cores of two AuNPs was $88 \AA$ and the protein was placed closely to the AuNP surface in the middle. To study the aggregation of proteins and Au flat surfaces, the distance between two carboxyl-terminated SAMs covered Au surfaces is $40 \AA$, and the distance between the cores of two proteins is $\sim 48.5 \AA$ with proteins in the middle of the Au surfaces. Atomeye was used for the visualization and image generation of all atomistic configurations. 


\section{CHAPTER 4 RESULTS AND DICUSSION}

\subsection{Model Validation}

SAMs formed from alkanethiol chains on Au surfaces have been used as model systems in many past studies on the interaction of proteins with surface ${ }^{122}$. The model and force field used in this research was also validated by systematically studying the adsorption of SAMs made of alkanethiol chains on Au (111) (110) and (100) surfaces and spherical AuNP, which can be compared with previous experimental observations and MD simulations. Overall our results showed that the crystal structure of the Au surface dramatically influences the packing of the SAMs, which is consistent with previous findings ${ }^{37,123}$.

We first used a simple single-chain model to study the organization of SAMs on Au surface and used the tilt angle of the chain as a key parameter to describe the SAMs behavior. Figure 4-1 (a) shows that at $300 \mathrm{~K}$, the tilt angle of a single alkanethiol chain on $\mathrm{Au}(111)$ is stabilized around $30^{\circ}$ after $1 \mathrm{~ns}$. We have also studied the effect of temperature on the structure of the methyl-terminated SAMs with the number of chains varied from 1 to 56 (7 chains in the $\mathrm{x}$ direction and 8 chains in the y direction). As shown in Fig. 4-1 (b), when the temperature was increased to $600 \mathrm{~K}$, the average tilt angle of the chain decreased to only around $10^{\circ}$. This is consistent with an earlier experimental study ${ }^{124}$ that the tilt angle of the alkanethiol chain on $\mathrm{Au}$ (111) decreased from $\sim 30^{\circ}$ to $\sim 8^{\circ}$ when the temperature increased from $300 \mathrm{~K}$ to $600 \mathrm{~K}$. Figure 4-1 (c) and (d) show the equilibrated model with 56 alkanethiol chains on Au (111) surface, which was consistent with the single chain model in terms of tilt angle and temperature effects. This result agreed very well with the previous findings ${ }^{125}$, which confirms the validity of the model and force fields used in our study. 

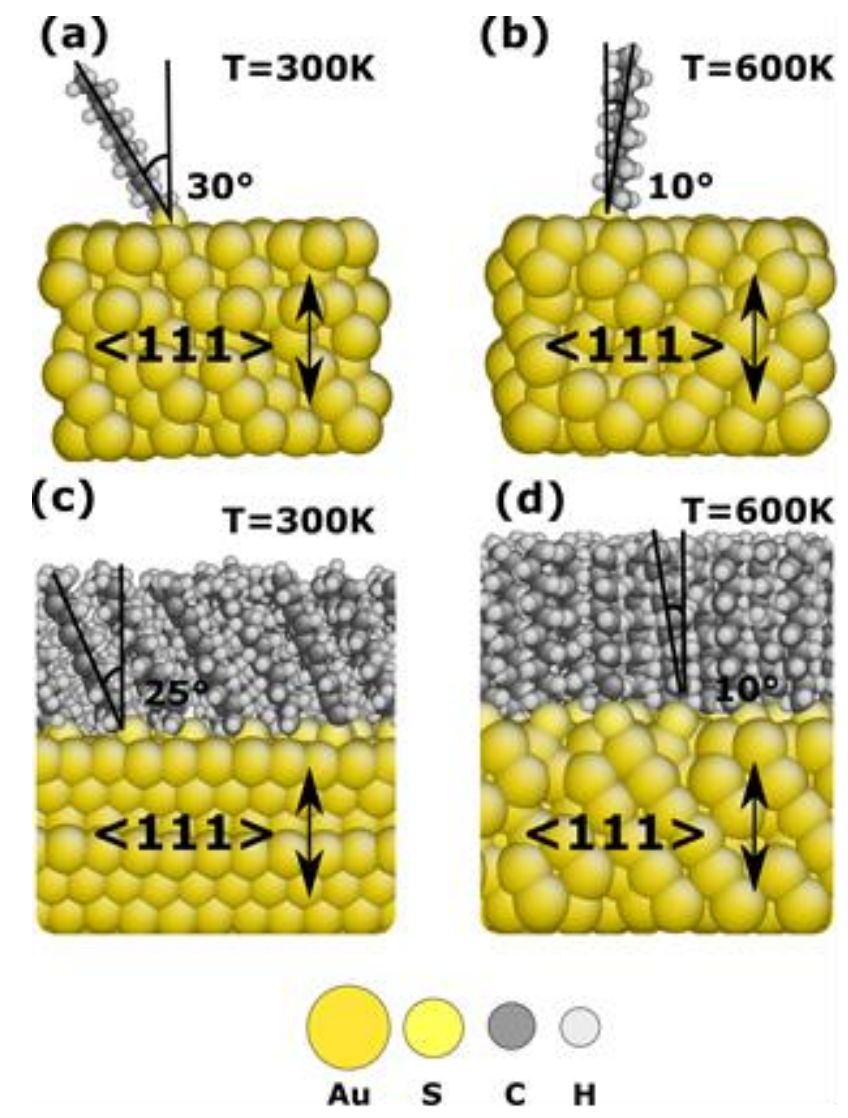

Figure 4-1. Equilibrated structure of SAMs on Au (111) surface from our MD simulations with one single alkanethiol chain at (a) $300 \mathrm{~K}$ and (b) $600 \mathrm{~K}$ and multiple alkanethiol chains at (c) $300 \mathrm{~K}$ and (d) $600 \mathrm{~K}$, respectively.

Then we studied the adsorption of SAMs with methyl functional group on the spherical AuNP at 300K. Figure 4-2 (a) shows the initial configuration of SAMs and AuNP. The interval distance between alkanethiol chains is $4.32 \AA$ in the $\mathrm{x}$ direction and $4.988 \AA$ in the $\mathrm{y}$ direction. And the SAMs were arranged $3 \AA$ away from the AuNP surface at the beginning. Figure 4-2 (b) shows that SAMs have been adsorbed on the spherical AuNP surface after 1ns, which also shows the accuracy of our force field. 


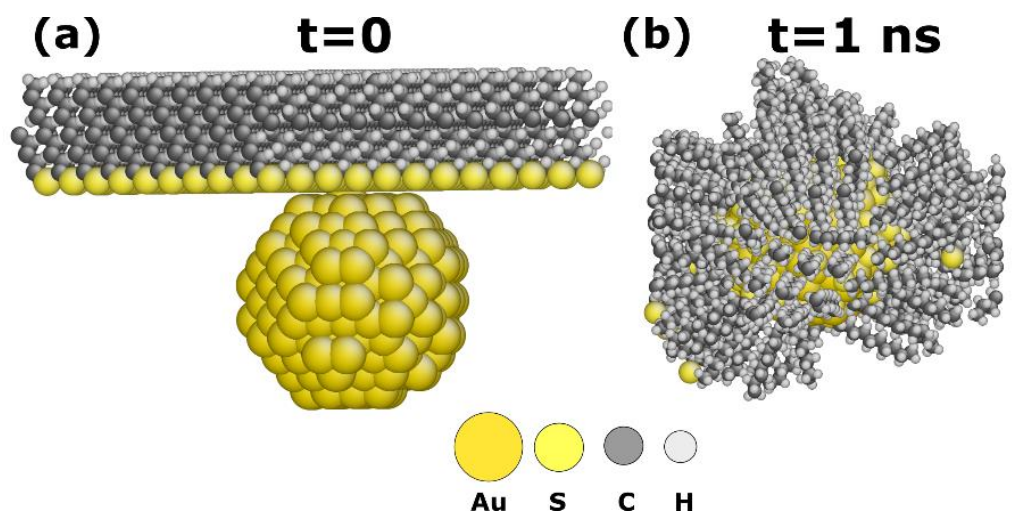

Figure 4-2. The adsorption of $\mathrm{CH}_{3}-\mathrm{SAMs}$ on spherical AuNPs at $300 \mathrm{~K}$.

\subsection{Influence of Au Surface on SAMs Formation}

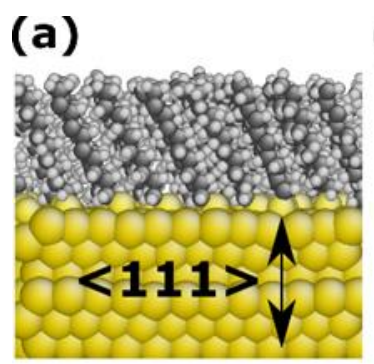

(b)
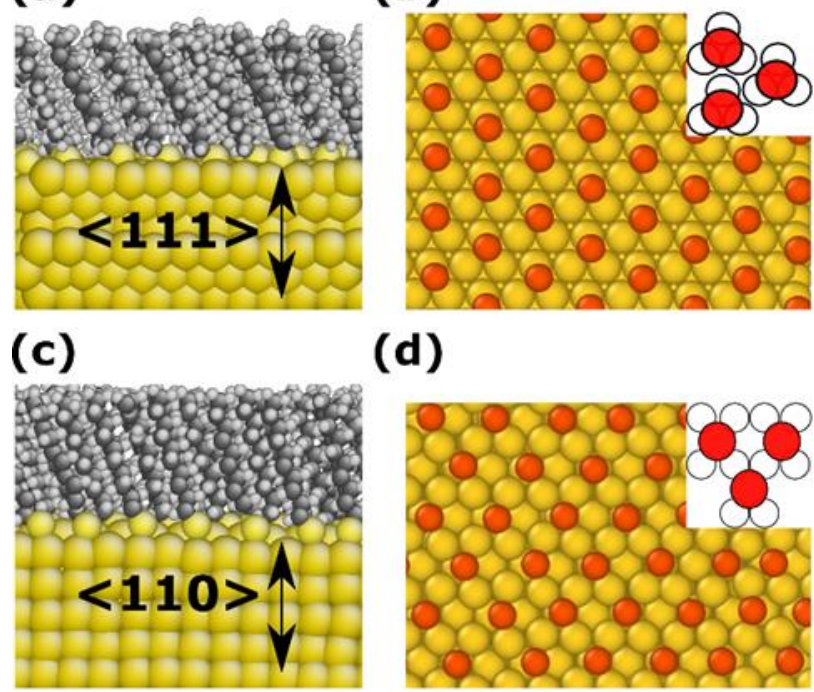

(d)

(e)

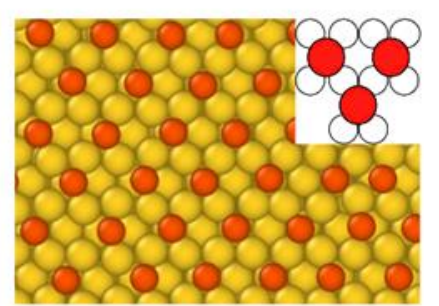

(f)
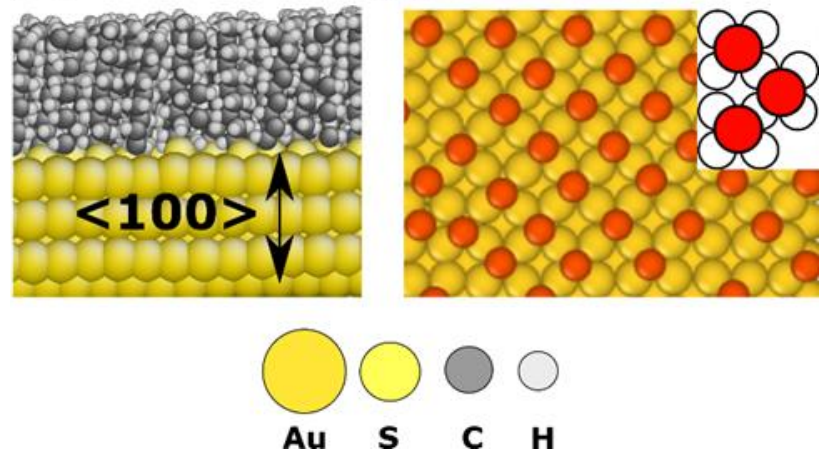

Figure 4-3. Side view of the equilibrium SAMs structures on (a) Au (111), (c) Au (110) and (e) $\mathrm{Au}$ (100) at $300 \mathrm{~K}$ and schematic diagram of S atoms on (b) Au (111), (d) Au (110) and (f) Au (100) at 300K. S atoms are changed in (b), (d) and (f) to red color for better view. 
The tilt angles and structure of SAMs with 56 chains on Au (111) (110) and (100) surfaces at $300 \mathrm{~K}$ were also studied and compared with experimental studies ${ }^{42}$. The results of $\mathrm{Au}(111)$ surface are also included here to facilitate comparison with the $\mathrm{Au}(110)$ and $\mathrm{Au}(100)$ systems. Figure 4-3 (a) shows an average of tilt angle around $25^{\circ}$ at $300 \mathrm{~K}$ on $\mathrm{Au}$ (111) surface, which agrees very well with the experimental tilt angle of $20^{\circ}$ to $40^{\circ}$ on $\mathrm{Au}(111)^{125 a}$. Figure 4-3 (b) shows a schematic diagram of the $\mathrm{S}$ atoms on $\mathrm{Au}$ (111) surface after running the simulation for 100 ps. Here the $S$ atoms are shown in red for better view. It can be seen that the $S$ atoms are bonded to the triple hollow sites of the Au (111) substrate lattice and form a commensurate ( $\sqrt{ }$ $3 \mathrm{x} \sqrt{ } 3)^{\mathrm{R}} 30^{\circ} \quad(\mathrm{R}=$ rotated) structure. This lattice presents distances of $4.988 \AA$ between the $\mathrm{S}$ heads of nearest neighbor molecules. This result again agreed very well with the previous experimental findings ${ }^{41}$, which further confirms the validity of the model and force fields used in our study.

In contrast, Figure 4-3 (c) shows that the average tilt angle of SAMs is around $35^{\circ}$ at 300 $\mathrm{K}$ on $\mathrm{Au}$ (110) surface with a rectangular unit lattice. Furthermore, Figure 4-3 (d) shows a schematic diagram of the packing of $\mathrm{S}$ atoms on $\mathrm{Au}(110)$ surface. There is a little distortion of S pattern on Au surface, but the tendency is that $\mathrm{S}$ atoms are bonded to the rectangular hollow sites and form a commensurate $\mathrm{c}(2 \times 2)$ structure with respect to the substrate $\mathrm{Au}(110)$ lattice. This result agreed very well with the experimental result shown by the low energy electro diffraction (LEED) pattern ${ }^{37}$. On the other hand, Figure 4-3 (e) shows the SAMs on Au (100) surface with a tilt angle around $10^{\circ}$ and Figure 4-3 (f) further indicates that the preferred adsorption of alkanethiol chains on Au (100) appears to make sulfur atoms organized as a c (2 $\times 2$ ) over layer again. Here the sulfur atoms are bonded to the rectangular hollow sites and the average distance between sulfur atoms are $5.765 \AA$. Although there are no previous reports on 
the structure of SAMs and the packing of sulfur atoms on Au (100) surface, our simulation results clearly show that the adsorption of alkanethiol chains on Au surface is strongly dependent on the structure of Au surface, and this assembly is dominated by the energetics of Au-sulfur bonding. The effect of chain-chain attraction is also important but lesser contributor to the assembly process.
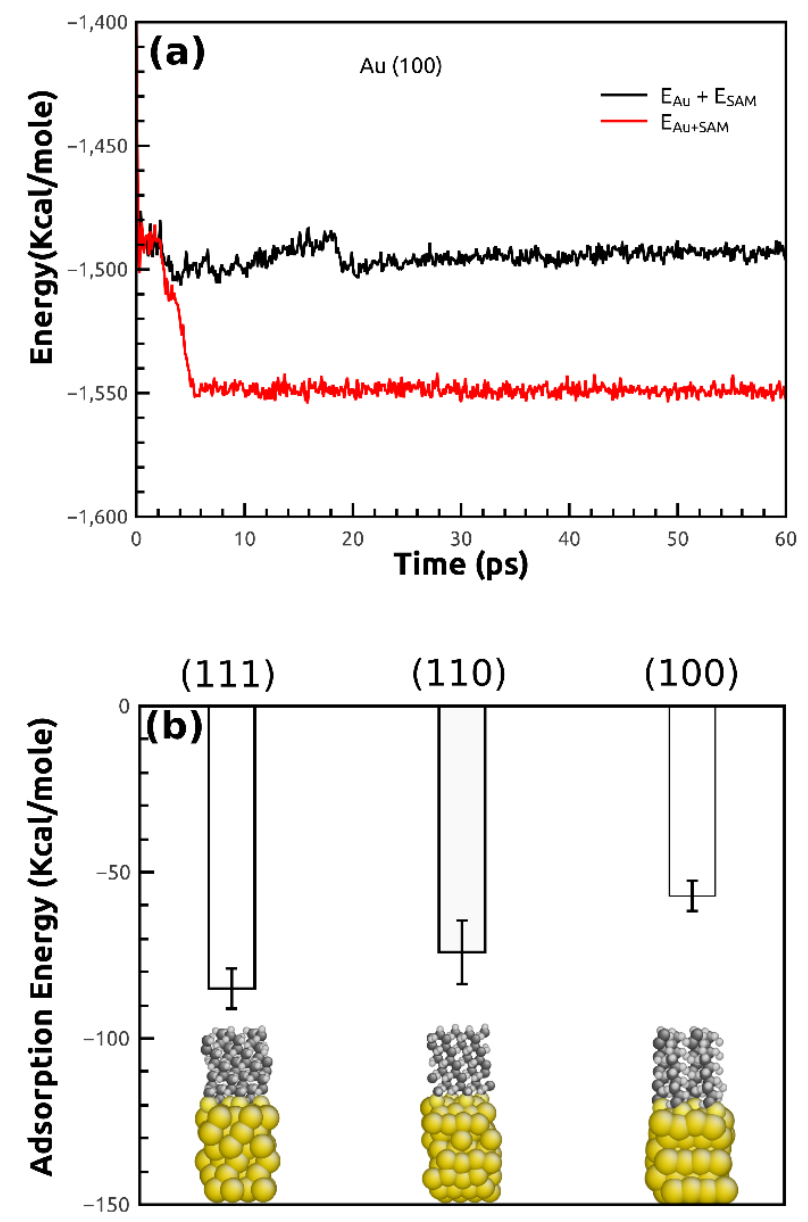

Figure 4-4. (a) The energy of a single alkanethiol chain on (100) Au surface before (black line) and after (red line) the adsorption. (b) The adsorption energy of a single alkanethiol chain on Au (111), (110) and (100) surfaces.

It has been proposed by Gagner et al. ${ }^{121}$ that the different atomic structures on Au surface may exert an influence on the adsorption of proteins because of their different surface energy. 
However, it is difficult to discern how the surface energy and crystal-face structure may contribute to adsorbed protein binding, structure and function by experimental methods at present. Additionally, while the difference in surface energy between $\mathrm{Au}(111),(110)$, and (100), e.g., $\gamma(111)<\gamma(100)<\gamma(110)^{126}$ has been well documented, the adsorption energy of SAMs on Au surface, however, has not yet been reported. Since the adsorption of proteins on AuNPs actually occurred through SAM, e.g., AuNP-SAMs-Protein, and the interaction between SAMs and protein should be more or less the same regardless the Au surface, it is important to investigate the adsorption energy of SAMs on different Au surfaces. Figure 4-4 (a) showed the energy of a single alkanethiol chain and Au (100) surface in black line, and the energy of combined system of $\mathrm{Au}(100)$ and chain after the adsorption in red line. It was found that the adsorption energy of an individual chain is average $-57 \pm 4 \mathrm{Kcal} / \mathrm{mole}$ on $\mathrm{Au}(100)$ surface, -74 $\pm 9 \mathrm{Kcal} / \mathrm{mole}$ on $\mathrm{Au}(110)$ surface, and $-85 \pm 6 \mathrm{Kcal} / \mathrm{mole}$ on $\mathrm{Au}$ (111) surface, which indicates the lowest adsorption energy on Au (111) surface, as shown in Figure 4-4 (b). This trend agreed very well with the experimental observations by Gagner et al. ${ }^{121}$ that $\mathrm{Au}$ (111) surface tends to have a relatively higher coverage of protein than other Au surfaces.

\subsection{Influence of Alkanethiol Chain Length on SAMs Formation}

Simulations of the adsorption of menthol terminated SAMs with different chain length on flat $\mathrm{Au}$ (111) surface were also performed to study the influence of chain length on the tilt angles of SAMs. In my study, the SAMs with 5 carbons and 20 carbons as the backbone have been studied at $300 \mathrm{~K}$. 


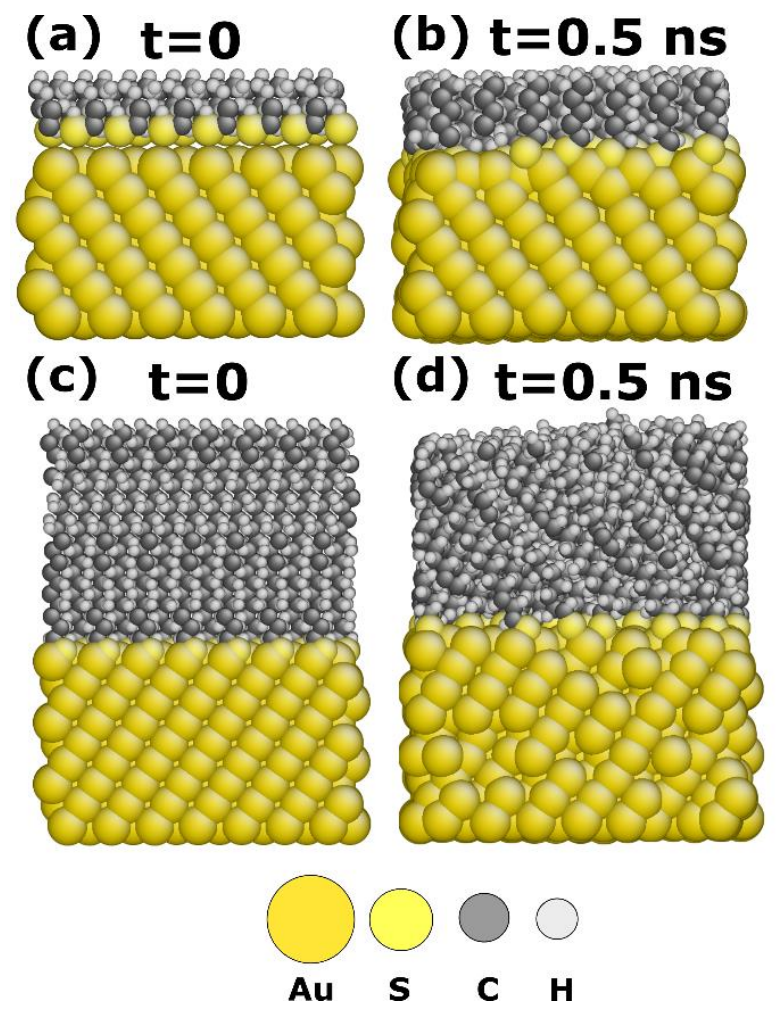

Figure 4-5. The influence of chain length on SAMs formation on $\mathrm{Au}(111)$ surface at $300 \mathrm{~K}$.

Figure 4-5 (a) and (c) show the initial positions of alkanethiol chains with 5 and 20 carbons respectively as backbone on the $\mathrm{Au}$ (111) surface at $300 \mathrm{~K}$. The interval distance between alkanethiol chains is also $4.32 \AA$ in the $\mathrm{x}$ direction and $4.988 \AA$ in the y direction on $\mathrm{Au}$ (111) surface. Figure 4-5 (b) and (d) show the final formation of SAMs on Au (111) after running the simulation for 0.5 ns. Compare Figure 4-5 (a) with (b), we did not see an obvious tilt angle of SAMs with 5 carbons. However, compare Figure 4-5 (c) and (d), the SAMs with 20 carbon atoms as the backbone have a more obvious tilt angle on $\mathrm{Au}$ (111) surface. This might be owing to the fact that the chain-chain interactions are increasingly important with the increase of the chain length and certain relative positions of the chains with respect to each other would be preferred. 


\subsection{Influence of Temperature on SAMs Arrangement on Spherical AuNPs}

Simulations of the adsorption of carboxyl terminated SAMs on spherical AuNPs were also performed to study the influence of curvature of AuNPs on the packing and arrangement of SAMs under various thermodynamics conditions. Figure 4-6 shows snapshots of the equilibrium structures of carboxyl terminated alkanethiol at different temperatures on a spherical AuNP with a diameter of $4 \mathrm{~nm}$. We observed that as the temperature was increased, the SAMs tended to distribute on the AuNP surface more evenly. At 300k in Figure 4-6 (a), the alkanethiol chains are tilted and ordered. While at higher temperatures, i.e., $600 \mathrm{~K}$ in Figure 46 (b) and 900K in Figure 4-6 (c), SAMs are more even on the surfaces. This results agreed very well with the studies done by Pradip et al. ${ }^{103 a}$ even though in their studies a united-atom model of SAMs with methyl tail groups was used.
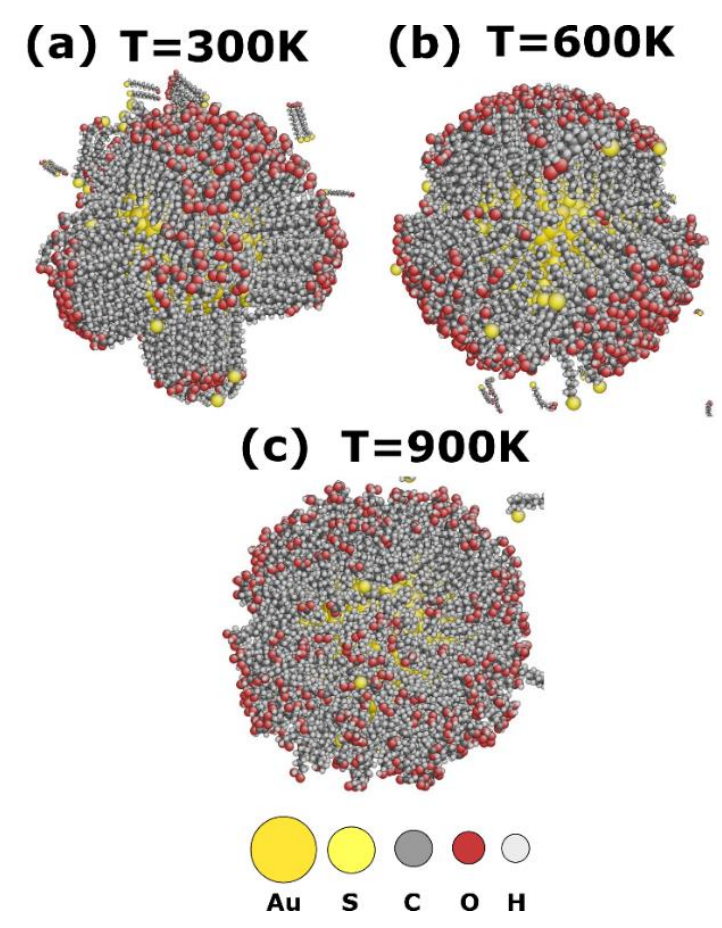

Figure 4-6. Snapshots of SAMs on spherical AuNP surfaces at different temperatures at (a) 300K, (b) 600K, (c) $900 \mathrm{~K}$. 
It needs to be noted that even though the polarization effect of Au surface was not fully considered in this work by using an EAM potential, the results in this work agreed very well with previous experimental studies, e.g., regarding the tilt angles and the sulfur atoms arrangement of SAMs on both the Au flat and spherical surfaces, showing the accuracy of using EAM potential for modeling Au with biomolecules. This is also one of the main purposes in this study, i.e., to introduce EAM potential in the modeling of metal with bimolecular system. The development of a proper potential is nontrivial. If EAM potential can yield consistent result with experimental observations and satisfactory accuracy, the rich library of EAM potentials may also be used to model other metals in bio-systems

\subsection{Influences of SAMs Terminal Functional Group on Protein Adsorption}

Since $\mathrm{Au}$ (111) surface has the lowest adsorption energy for alkanethiol chains, Au (111) was used in the following study on the adsorption of Lyz protein on Au surfaces. In this study, SAMs of alkanethiol chains terminated with both methyl and carboxyl functional groups were used.

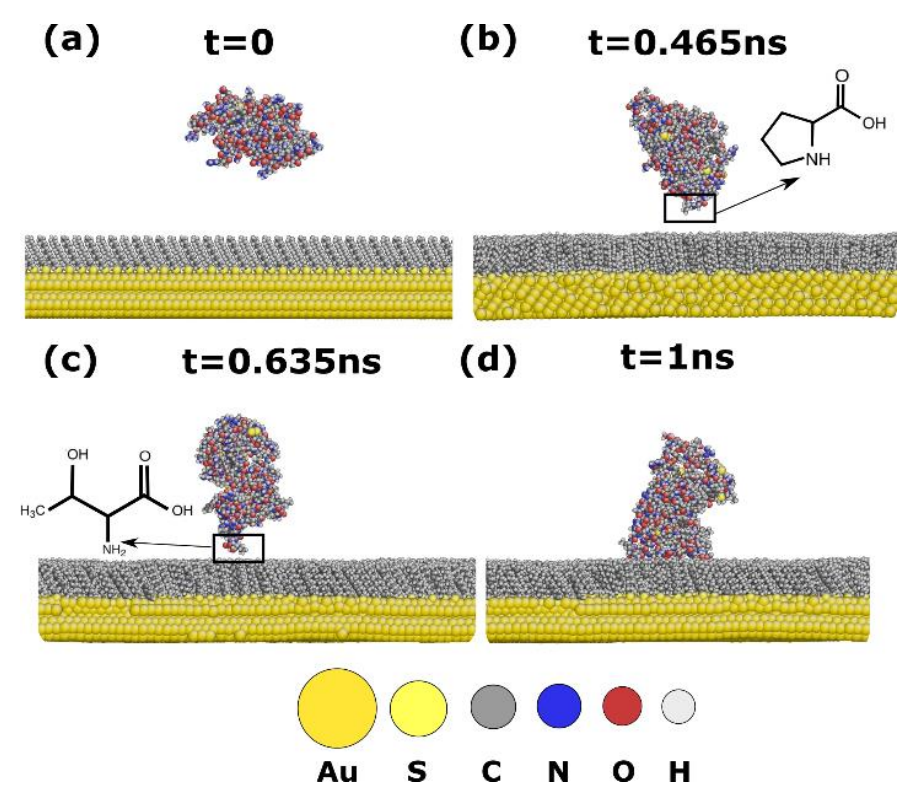


Figure 4-7. Snapshot of the interaction between protein and methyl-terminated SAMs.

Figure 4-7 shows the adsorption of Lyz protein on the methyl-terminated SAMs surface at 300 K. All the simulations have been run for three times with different initial position of the protein as discussed in the Methodology section. Figure 4-7 (a) shows a representative initial configuration of the system in which the SAMs were $5 \AA$ away from the Au (111) surface and a Lyz molecule was $24 \AA$ away from the methyl-terminated SAMs. During the simulation we observed that the protein moved closer to the hydrophobic surface with dramatic rotation to adjust the exposed amino acids to the hydrophobic surfaces. After $0.465 \mathrm{~ns}$, as shown in Figure 4-7 (b), attractive interactions occurred between the protein and the methyl-terminated SAMs. By zooming in the adsorption sites, the amino acid exposed to the hydrophobic surface was identified to be proline, a type of less hydrophobic amino acid. However, the protein was not stable and still kept rotating to adjust the configuration. After $0.635 \mathrm{~ns}$, different amino acid stretched out and exposed to the hydrophobic surface as shown in Figure 4-7 (c). By zooming the adsorption site in Figure 4-7 (c), it was found that the amino acid of the Lyz attached to the hydrophobic methyl-terminated SAMs is leucine, a strong hydrophobic amino acid. At this time the rotation movement was reduced with the leucine amino acid attached to the hydrophobic surface stably after $1 \mathrm{~ns}$ as shown in Figure 4-7 (d).

It is known that hydrophobic SAMs enhance protein adsorption ${ }^{127}$. Therefore, when a protein is brought closer to the hydrophobic methyl- terminated SAMs without the hydration layer, the hydrophobic interactions between the protein and the methyl-terminated SAMs may cause the protein to expose its internal hydrophobic residues to the surface, leading to protein adsorption. Furthermore, our results agreed very well with the studies by Grzegorz et al. ${ }^{128}$ showing that for the hydrophobic Au, adsorption events of a small protein were driven by attraction to the strongest binding amino acids. Since the leucine amino acid is more 
hydrophobic than proline amino acid, the protein kept rotating while approaching to the surface and was finally adsorbed on the hydrophobic surface through the more hydrophobic amino acid.

Although some simulation results showed that the total interfacial force may mainly come from the hydration water ${ }^{104 a}$, not from the SAMs surface, we do find that the properties of SAMs have strong influence on the adsorption of protein. To explore the effect of SAMs morphology on the adsorption of protein, the interaction between the Lyz protein and charged self-assembled monolayers (SAMs) terminating with carboxyl functional groups on Au (111) surface has also been studied.

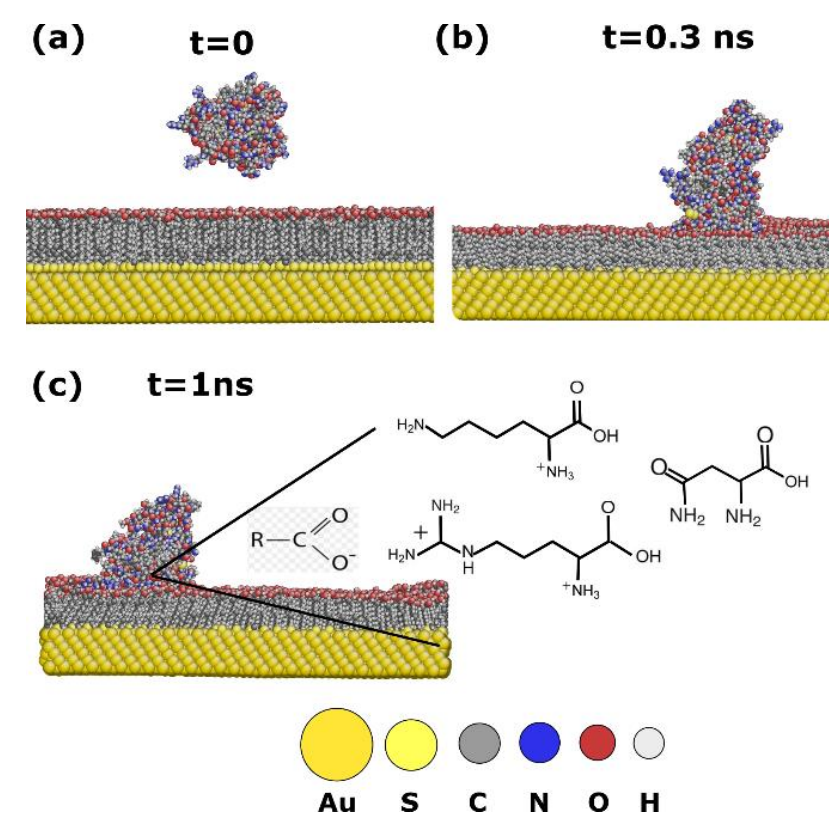

Figure 4-8. The electrostatic interactions occurred between Carboxyl-terminated SAMs and protein amino acids.

Figure 4-8 (a) shows the initial configuration of the system with the SAMs $5 \AA$ away from the $\mathrm{Au}$ (111) surface and a single lysozyme protein molecule $10 \AA$ away from the carboxylterminated SAMs. The interactions between the protein's residues and the carboxyl-terminated SAMs surface were different from the methyl terminated SAMs surface. Figure 4-8 (b) shows that at $0.3 \mathrm{~ns}$, the protein was attached to the carboxyl group-terminated SAM. With the increase 
of time, we observed that the protein was still attached to the Au surface firmly, as shown in Figure 4-8 (c) at $1 \mathrm{~ns}$. We examined the adsorption process carefully in order to identify the types of interactions responsible for the protein's amino acid being preferentially attracted to the surface. It was found that the amino acids of Lyz attached to the carboxyl-terminated SAMs are lysine and arginine, which are two types of amino acid with positive charges, and the asparagine, which is a type of neutral charged amino acid. Therefore, one of the primary bonding for this system was the electrostatic interaction between positively charged residues such as arginine and lysine and the carboxyl groups of the SAMs. The interaction may also be enhanced by the redistribution of charges of the neutral charged asparagine, so that they can be attached to the carboxyl functional group. The detailed interaction occurred between the amino acids and these SAMs surfaces are shown in Figure 4-8 (c).

In order to confirm our simulation results, we rotated the protein by $180^{\circ}$ and we found that after the rotation, the protein could be adsorbed on the Au surface more effectively for exposing different functional groups to the SAMs surface, which agreed with the studies by Lopez et. al ${ }^{129}$ who showed that the adsorption process of protein also depends on the protein orientation. Furthermore, if the temperature was increased to $600 \mathrm{~K}$, the adsorption process of protein was faster than that at $300 \mathrm{~K}$.

While all these simulations were performed considering the implicit water environment, we also run the simulation without the solvent for comparison. The results showed that the adsorption of protein was enhanced in the water solvent on the methyl terminated SAMs surface, which may be owing to the density and polarization of water. Without the water solvent, the protein could still be adsorbed on the methyl terminated SAMs surface but detached after around 20 ps, which showed the molecular dynamics simulations depend on the details of the 
force field. Regarding the adsorption of protein on the carboxyl-terminated SAMs Au surface, the adsorption was slightly reduced with the solvent comparing the one without the solvent. But in all the simulations, the conclusions of SAMs on Au surface are the same, showing the negligible influence of polarization for SAMs coating on Au surface.

The adsorption energy of protein on both carboxyl-terminated SAMs and methyl terminated SAMs surfaces was later calculated using the method discussed in the Methodology section. Our results showed that the adsorption energy on the methyl-terminated and carboxylterminated SAM surface based on the initial configurations shown in Figures 4-7 and 4-8 are around $-240 \mathrm{Kcal} / \mathrm{mole}$ and $-291 \mathrm{Kcal} /$ mole respectively, which indicate that the adsorption was stronger on the carboxyl-terminated than methyl-terminated SAMs. Therefore, we concluded that the carboxyl-terminated SAMs can adsorb proteins more effectively comparing with the methyl terminated SAMs. Yun et al. ${ }^{130}$ are studied the energy landscape of the lysozyme adsorbed on a negatively charged surface as a function of the protein orientation. From their studies, the lowest adsorption energy was around $-102.8 \mathrm{Kcal} / \mathrm{mole}$, which is on the same order of magnitude of our results. Since they used the Monte Carlo simulation rather than MD on a surface without the coverage of SAMs, our results are fairly comparable with theirs. While those energies may change with different initial positions or orientations of the protein molecule, it shows that the carboxyl group-terminated SAMs are more effective to absorb Lyz protein than the methyl-terminated SAMs. This finding is again consistent with the experiments performed by Gagner et al. ${ }^{121}$ who intentionally included the aqueous-organic ligand exchange procedure to replace the methyl group by carboxyl group (i.e., from dodecanethiol (DDT) to 16-mercaptohexadecanoic acid 90\% (MHDA)) in the SAMs of their Au NPs to enhance the protein adsorption. 


\subsection{Formation of AuNP-Protein Conjugate}

In order to explore the atomistic mechanisms of the formation and aggregation of proteinAuNP conjugates as observed in experiments ${ }^{121}$, we first studied the interactions between two standalone Lyz protein molecules and the interactions between two standalone Au (111) surfaces covered with carboxyl-terminated SAMs. Our results showed that there is no strong interaction between two standalone protein molecules as indicated in Figure 4-9 (a) and (b). Figure 4-9 (a) shows the initial configuration of two Lyz molecules which are close enough to each other and can move freely in a big simulation box. The two protein molecules did not tend to move closer to each other and Figure 4-9 (b) shows the final position of them. Our results also showed no obvious attraction between two standalone Au surfaces covered with carboxylterminated SAMs. As shown in Figure 4-9 (c) and (d), the two Au (111) surfaces remained almost the same distance as initial configuration after running the simulation for $1 \mathrm{~ns}$.

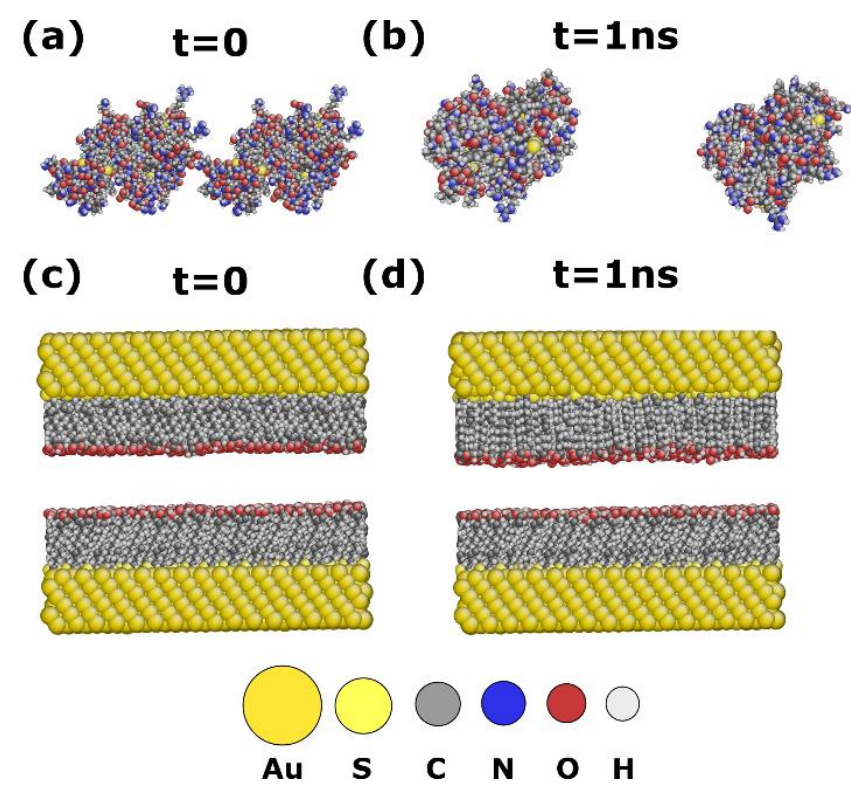

Figure 4-9. (a) and (b) The interaction between two Lysozyme protein molecules. (c) and (d) The interaction between two $\mathrm{Au}$ (111) surfaces covered with carboxyl-terminated SAMs. 

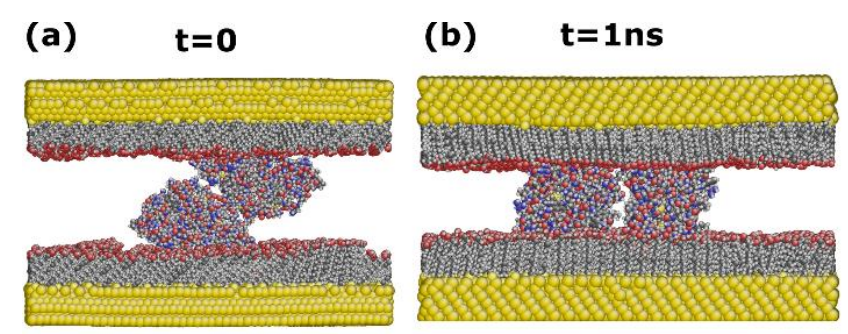

(c)

(d)

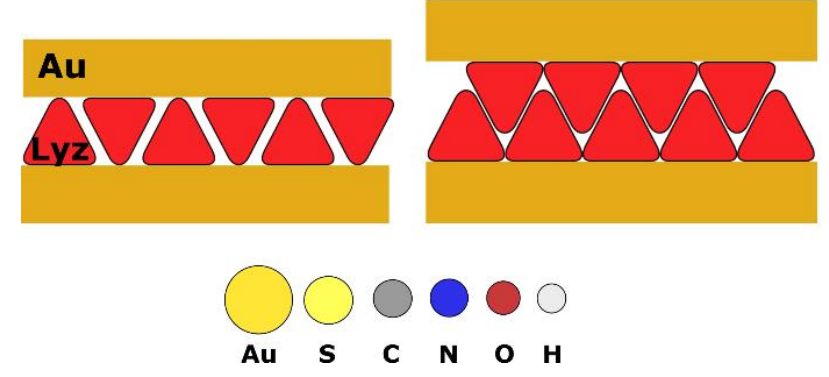

Figure 4-10. (a) and (b) The adsorption between Lyz protein and two Au surfaces covered with carboxylterminated SAMs. (c) and (d) Schematic of Lyz-AuNP conjugate formation proposed in my research and the previous study ${ }^{121}$.

Since there is no strong attraction between standalone proteins or Au surfaces, the formation of protein-AuNP conjugate and aggregation must be owing to the interaction between protein and the SAMs covered Au surfaces. We tested our hypothesis by placing a Lyz protein between two SAMs covered Au (111) surfaces, as shown in Figure 4-10 (a). Figure 4-10 (b) shows that after 1 ns, strong adsorption occurred between the protein molecule and SAMs on both Au surfaces which formed the Lyz-AuNP aggregation. Based on this observation, we propose a schematic model in Figure 4-10 (c) to show the formation of AuNP-protein aggregation, where the rectangles represent AuNPs that are covered with SAMs, and the red triangles represents Lyz protein molecules. We propose in this model that the formation of Lyz-AuNP conjugates is mainly due to protein- SAMs surface interaction. This model is slightly different from that shown in Figure 4-10 (d), which is proposed by Gagner et al ${ }^{121}$ based on their experiments. In their model, it seems to suggest that the formation of Lyz-AuNP conjugates is largely due to strong protein-protein interaction between the individual AuNPs, which is not observed in our MD simulations (Figure 4-10 (d)). 


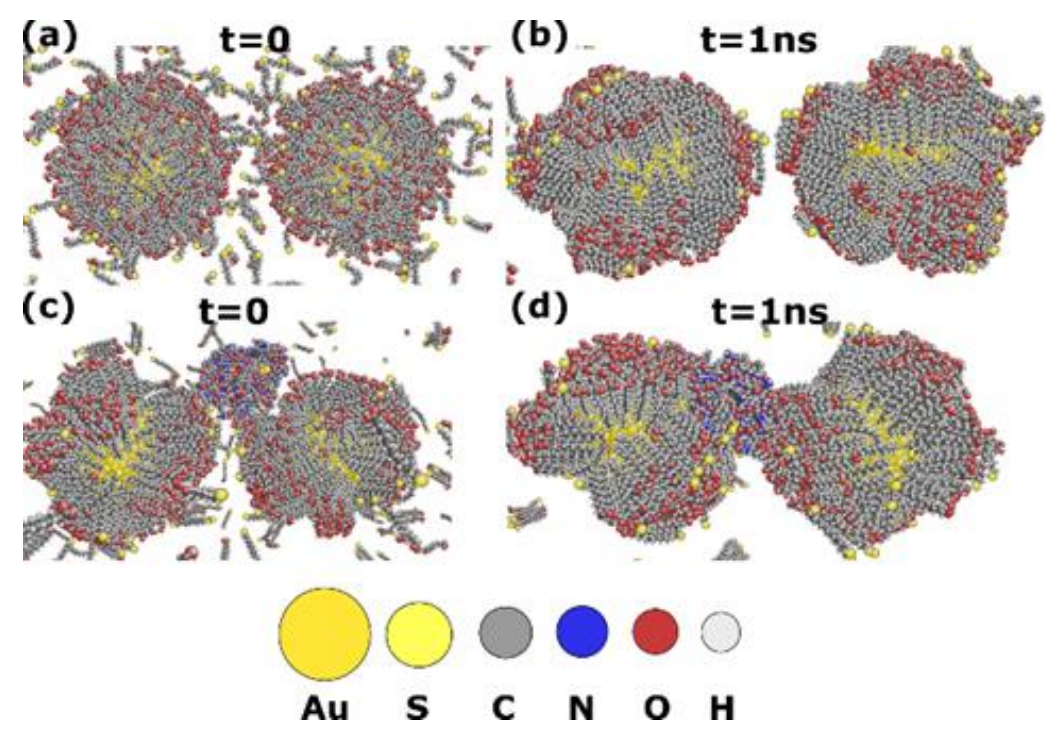

Figure 4-11. (a) and (b) The interaction between two carboxyl-terminated SAMs covered AuNPs. (c) and (d) The interaction between two spherical AuNPs covered with carboxyl-terminated SAMs and the protein.

In order to further confirm the formation mechanism of AuNP-protein conjugate proposed in Figure 4-10 (c), we repeated the simulations with spherical AuNPs. First we studied the interaction between two AuNPs. As shown in Figure 4-11 (a) and (b), after 1 ns, the two spherical AuNPs did not attach to each other. Although Thompson et al. ${ }^{131}$ concluded in their studies that the collective driving force was very large to aggregate the large $30 \mathrm{~nm}$-diameter NPs, we did not observe any strong attraction between two free AuNPs as by Thompson et al. ${ }^{131}$. However, their studies pointed out that this very large inter-particle cohesive force is due to the large number of chains at the $30 \mathrm{~nm}$-diameter nanoparticles interface. Since our simulation is based on the spherical AuNP with only 4nm diameter, and it is the carboxyl-terminated SAMs covered AuNPs rather than the hydrophobic SAMs, the different behavior between two AuNPs might be owing to the difference in the models used in the respective study. Additionally, Figure 4-11 (c) and (d) showed the interaction of a Lyz protein molecule with two AuNPs. It was found that the amino acids of the protein that have been adsorbed on the surface were arginine and lysine owing to the electrostatic interaction, showing the same mechanism was with the flat $\mathrm{Au}$ 
surface. Since a flat Au surface can be essentially treated as an AuNP with zero curvature or infinitely large radius, our results imply that the size or the curvature of the AuNP will not influence the fundamental adsorption process of protein. 


\section{CHAPTER 5 CONCLUSIONS AND FUTURE WORK}

\subsection{Conclusions}

In summary, MD simulation has been applied in this research with an all-atom model to study:

a). The arrangement of SAMs on AuNP surfaces, including:

- The influence of AuNP morphologies on the arrangement of SAMs.

- The adsorption energy of SAMs on flat Au (111), (110) and (100) surfaces.

- The influence of SAM chain length on the SAMs formation.

- The influence of temperatures on the arrangement of SAMs on spherical AuNPs.

b). The interaction between SAMs covered AuNPs and Lyz proteins, including:

- The influences of SAMs terminal functional groups on protein adsorption on $\mathrm{Au}$ surfaces.

- The mechanism of the formation of the protein-AuNP conjugates.

Based on these studies, the following conclusions can be made:

1. The accuracy of an all-atom model with Morse potential for Au-S interaction, LennardJones potential for proteins, SAMs and implicit water system, and the most importantly, the EAM potential for AuNPs has been approved. The accuracy of EAM potential in modeling of metal with bimolecular system sheds light on the future research on the metal-biosystem simulations.

2. The consistency of our simulation results with previous experimental results also shows the reliability of LAMMPS software in the study of biosystems, which has been considered to be difficult in previous research.

3. The $\mathrm{Au}$ surface morphologies dramatically influence the adsorption energy and 
arrangement of SAMs. It was found that SAMs have the lowest adsorption energy on flat $\mathrm{Au}(111)$ surface and form a commensurate $(\sqrt{ } 3 \mathrm{x} \sqrt{ } 3) \mathrm{R} 30^{\circ}$ on $\mathrm{Au}(111)$ surface at $300 \mathrm{~K}$.

4. The SAMs with longer chain length have larger tilt angles on $\mathrm{Au}(111)$ surface at $300 \mathrm{~K}$.

5. As the temperature was increased from $300 \mathrm{~K}$ to $900 \mathrm{~K}$, the SAMs tended to distribute on the spherical AuNP surface more evenly.

6. Proteins can be adsorbed on the AuNPs covered by methyl-terminated and carboxylterminated SAMs owing to the hydrophobic interaction and electrostatic interactions, respectively. Carboxyl-terminated SAMs are more effective to absorb proteins compared with the methyl-terminated SAMs.

7. The simulation studies confirmed that the formation of AuNP-protein conjugates is due to protein-COOH-SAMs interaction, not protein-protein interactions as suggested in previous studies.

\subsection{Future Work}

The future work of this study will be focused on:

1. Using explicit water molecules to study the interaction between proteins and AuNPs in water environment, and comparing the results with the current implicit water system.

2. Enlarging the size of the simulation system to consider the size effect.

3. Modifying the EAM potential for Au to take account the polarization effect.

One peer-reviewed journal publication and several conference presentations have been developed based on these results:

\section{Publications:}

- Aoran Wei, Chuang Deng, “Adsorption of Protein on a Au Surface Studied by All-Atom 
Atomistic Simulations", The Journal of Physical Chemistry C, 2016, 120 (24), pp 13103-13112.

\section{Conferences and Competitions:}

Manitoba Materials Conference, University of Manitoba, Winnipeg, Canada. May 10, 2016

- Aoran Wei, Chuang Deng, "The adsorption of protein on Au surface studied by allatom atomistic simulations".

251st American Chemical Society National Meeting \& Exposition, San Diego, California, U.S. March 13-17, 2016

- Aoran Wei, Chuang Deng, "The adsorption of protein on Au surface studied by all-atom atomistic simulations".

Mechanical Engineering Poster Competition, University of Manitoba, Winnipeg, Canada. November 03, 2015

- Aoran Wei, Chuang Deng, "Simulation of self-assembled monolayers on gold surface".

BiomedCon 2015, University of Manitoba, Winnipeg, Canada.

October 30, 2015

- Aoran Wei, Chuang Deng, "Simulation of self-assembled monolayers on gold surface".

Manitoba Materials Conference 2015, University of Manitoba, Winnipeg, Canada. May 12, 2015

- Aoran Wei, Chuang Deng, "Simulation of self-assembled monolayers on gold surface

\section{Awards:}

- Best Master's Student Poster during the Manitoba Materials Conference

- 3rd place - Mechanical Engineering Poster Competition, University of Manitoba 2015 


\section{REFERENCE}

1. Wagner, V.; Dullaart, A.; Bock, A.-K.; Zweck, A., The emerging nanomedicine landscape. Nature biotechnology 2006, 24 (10), 1211-1217.

2. Faraji, M.; Yamini, Y.; Rezaee, M., Magnetic nanoparticles: synthesis, stabilization, functionalization, characterization, and applications. Journal of the Iranian Chemical Society 2010, 7 (1), 1-37.

3. Boisselier, E.; Astruc, D., Gold nanoparticles in nanomedicine: preparations, imaging, diagnostics, therapies and toxicity. Chemical society reviews 2009, 38 (6), 1759-1782.

4. Farokhzad, O. C.; Langer, R., Nanomedicine: developing smarter therapeutic and diagnostic modalities. Advanced drug delivery reviews 2006, 58 (14), 1456-1459.

5. Gref, R.; Minamitake, Y.; Peracchia, M. T.; Trubetskoy, V.; Torchilin, V.; Langer, R., Biodegradable long-circulating polymeric nanospheres. Science 1994, 263 (5153), 1600-1603.

6. Bruchez, M.; Moronne, M.; Gin, P.; Weiss, S.; Alivisatos, A. P., Semiconductor nanocrystals as fluorescent biological labels. science 1998, 281 (5385), 2013-2016.

7. Cui, Y.; Wei, Q.; Park, H.; Lieber, C. M., Nanowire nanosensors for highly sensitive and selective detection of biological and chemical species. Science 2001, 293 (5533), 12891292.

8. Freitas, R. A., What is nanomedicine? Nanomedicine: Nanotechnology, Biology and Medicine 2005, 1 (1), 2-9.

9. Saptarshi, S. R.; Duschl, A.; Lopata, A. L., Interaction of nanoparticles with proteins: relation to bio-reactivity of the nanoparticle. Journal of nanobiotechnology 2013, 11 (1), 1. 10. Rana, S.; Bajaj, A.; Mout, R.; Rotello, V. M., Monolayer coated gold nanoparticles for delivery applications. Advanced drug delivery reviews 2012, 64 (2), 200-216.

11. Fan, J.; Chen, S.; Gao, Y., Coating gold nanoparticles with peptide molecules via a peptide elongation approach. Colloids and Surfaces B: Biointerfaces 2003, 28 (2), 199-207. 12. Torchilin, V., Tumor delivery of macromolecular drugs based on the EPR effect. Advanced drug delivery reviews 2011, 63 (3), 131-135.

13. Treuel, L.; Nienhaus, G. U., Toward a molecular understanding of nanoparticleprotein interactions. Biophysical Reviews 2012, 4 (2), 137-147.

14. Ding, Y.; Jiang, Z.; Saha, K.; Kim, C. S.; Kim, S. T.; Landis, R. F.; Rotello, V. M., Gold nanoparticles for nucleic acid delivery. Molecular Therapy 2014, 22 (6), 1075.

15. Nguyen, D. T.; Kim, D.-J.; Kim, K.-S., Controlled synthesis and biomolecular probe application of gold nanoparticles. Micron 2011, 42 (3), 207-227.

16. Parab, H.; Jung, C.; Woo, M.-A.; Park, H. G., An anisotropic snowflake-like structural assembly of polymer-capped gold nanoparticles. Journal of Nanoparticle Research 2011, 13 (5), 2173-2180.

17. Zhao, P.; Li, N.; Astruc, D., State of the art in gold nanoparticle synthesis.

Coordination Chemistry Reviews 2013, 257 (3), 638-665.

18. Turkevich, J.; Stevenson, P. C.; Hillier, J., A study of the nucleation and growth processes in the synthesis of colloidal gold. Discussions of the Faraday Society 1951, 11, 5575.

19. Frens, G., Controlled nucleation for the regulation of the particle size in monodisperse gold suspensions. Nature 1973, 241 (105), 20-22. 
20. Kimling, J.; Maier, M.; Okenve, B.; Kotaidis, V.; Ballot, H.; Plech, A., Turkevich method for gold nanoparticle synthesis revisited. The Journal of Physical Chemistry B 2006, 110 (32), 15700-15707.

21. Brown, K. R.; Fox, A. P.; Natan, M. J., Morphology-dependent electrochemistry of cytochrome $\mathrm{c}$ at $\mathrm{Au}$ colloid-modified $\mathrm{SnO} 2$ electrodes. Journal of the American Chemical Society 1996, 118 (5), 1154-1157.

22. Giersig, M.; Mulvaney, P., Preparation of ordered colloid monolayers by electrophoretic deposition. Langmuir 1993, 9 (12), 3408-3413.

23. Brust, M.; Walker, M.; Bethell, D.; Schiffrin, D. J.; Whyman, R., Synthesis of thiolderivatised gold nanoparticles in a two-phase liquid-liquid system. Journal of the Chemical Society, Chemical Communications 1994, (7), 801-802.

24. Templeton, A. C.; Wuelfing, W. P.; Murray, R. W., Monolayer-protected cluster molecules. Accounts of chemical research 2000, 33 (1), 27-36.

25. Yong, K.-T.; Swihart, M. T.; Ding, H.; Prasad, P. N., Preparation of gold nanoparticles and their applications in anisotropic nanoparticle synthesis and bioimaging. Plasmonics 2009, 4 (2), 79-93.

26. Aslam, M.; Fu, L.; Su, M.; Vijayamohanan, K.; Dravid, V. P., Novel one-step synthesis of amine-stabilized aqueous colloidal gold nanoparticles. Journal of materials Chemistry 2004, 14 (12), 1795-1797.

27. Jana, N. R.; Gearheart, L.; Murphy, C. J., Seeding growth for size control of 5-40 nm diameter gold nanoparticles. Langmuir 2001, 17 (22), 6782-6786.

28. Tabrizi, A.; Ayhan, F.; Ayhan, H., Gold nanoparticle synthesis and characterisation. Hacettepe journal of biology and chemistry 2009, 37 (3), 217-226.

29. Skala, M. C.; Crow, M. J.; Wax, A.; Izatt, J. A., Photothermal optical coherence tomography of epidermal growth factor receptor in live cells using immunotargeted gold nanospheres. Nano letters 2008, 8 (10), 3461-3467.

30. Liz-Marzán, L. M., Tailoring surface plasmons through the morphology and assembly of metal nanoparticles. Langmuir 2006, 22 (1), 32-41.

31. Sperling, R. A.; Gil, P. R.; Zhang, F.; Zanella, M.; Parak, W. J., Biological applications of gold nanoparticles. Chemical Society Reviews 2008, 37 (9), 1896-1908.

32. Barlow, S. M.; Raval, R., Complex organic molecules at metal surfaces: bonding, organisation and chirality. Surface Science Reports 2003, 50 (6), 201-341.

33. Love, J. C.; Estroff, L. A.; Kriebel, J. K.; Nuzzo, R. G.; Whitesides, G. M., Selfassembled monolayers of thiolates on metals as a form of nanotechnology. Chemical reviews 2005, 105 (4), 1103-1170.

34. Cho, S.-H.; Lee, Y.-U.; Lee, J.-S.; Jo, K.-M.; Kim, D.-H.; Kim, B.-S.; Han, M.-K., The Effect of Self Assembled Monolayer (SAM) on the Back Interface of a-IGZO TFT. ECS Transactions 2011, 35 (2), 353-359.

35. Aubin-Tam, M.-E.; Hamad-Schifferli, K., Structure and function of nanoparticleprotein conjugates. Biomedical Materials 2008, 3 (3), 034001.

36. Maksymovych, P.; Voznyy, O.; Dougherty, D. B.; Sorescu, D. C.; Yates, J. T., Gold adatom as a key structural component in self-assembled monolayers of organosulfur molecules on Au (111). Progress in Surface Science 2010, 85 (5), 206-240.

37. Strong, L.; Whitesides, G. M., Structures of self-assembled monolayer films of organosulfur compounds adsorbed on gold single crystals: electron diffraction studies. Langmuir 1988, 4 (3), 546-558. 
38. Kanis, D.; Mirkin, C. A.; McDevitt, J. T. Molecular Control Over the Interfacial Properties of High-T (c) Superconductors; DTIC Document: 2000.

39. Chidsey, C. E.; Liu, G. Y.; Rowntree, P.; Scoles, G., Molecular order at the surface of an organic monolayer studied by low energy helium diffraction. The Journal of chemical physics 1989, 91 (7), 4421-4423.

40. Whitesides, G. M.; Laibinis, P. E., Wet chemical approaches to the characterization of organic surfaces: self-assembled monolayers, wetting, and the physical-organic chemistry of the solid-liquid interface. Langmuir 1990, 6 (1), 87-96.

41. Fenter, P.; Eisenberger, P.; Liang, K., Chain-length dependence of the structures and phases of CH 3 (CH 2) n- 1 SH self-assembled on Au (111). Physical review letters 1993, 70 (16), 2447.

42. Camillone III, N.; Chidsey, C. E.; Liu, G. y.; Scoles, G., Substrate dependence of the surface structure and chain packing of docosyl mercaptan self-assembled on the (111),(110), and (100) faces of single crystal gold. The Journal of chemical physics 1993, 98 (5), 42344245.

43. Alkilany, A. M.; Murphy, C. J., Toxicity and cellular uptake of gold nanoparticles: what we have learned so far? Journal of nanoparticle research 2010, 12 (7), 2313-2333.

44. Goodman, C. M.; McCusker, C. D.; Yilmaz, T.; Rotello, V. M., Toxicity of gold nanoparticles functionalized with cationic and anionic side chains. Bioconjugate chemistry 2004, 15 (4), 897-900.

45. Patra, H. K.; Banerjee, S.; Chaudhuri, U.; Lahiri, P.; Dasgupta, A. K., Cell selective response to gold nanoparticles. Nanomedicine: Nanotechnology, Biology and Medicine 2007, 3 (2), 111-119.

46. Gagner, J. E.; Shrivastava, S.; Qian, X.; Dordick, J. S.; Siegel, R. W., Engineering nanomaterials for biomedical applications requires understanding the nano-bio interface: a perspective. The journal of physical chemistry letters 2012, 3 (21), 3149-3158.

47. Gagner, J. E.; Qian, X.; Lopez, M. M.; Dordick, J. S.; Siegel, R. W., Effect of gold nanoparticle structure on the conformation and function of adsorbed proteins. Biomaterials 2012, 33 (33), 8503-8516.

48. Lee, Y. K.; Choi, E.-J.; Webster, T. J.; Kim, S.-H.; Khang, D., Effect of the protein corona on nanoparticles for modulating cytotoxicity and immunotoxicity. Inter J Nanomed 2015, 10, 97-113.

49. Wang, P.; Wang, X.; Wang, L.; Hou, X.; Liu, W.; Chen, C., Interaction of gold nanoparticles with proteins and cells. Science and Technology of Advanced Materials 2016.

50. Yang, S. T.; Liu, Y.; Wang, Y. W.; Cao, A., Biosafety and bioapplication of nanomaterials by designing protein-nanoparticle interactions. Small 2013, 9 (9-10), 1635 1653.

51. Geoghegan, W. D.; Ackerman, G. A., Adsorption of horseradish peroxidase, ovomucoid and anti-immunoglobulin to colloidal gold for the indirect detection of concanavalin A, wheat germ agglutinin and goat anti-human immunoglobulin $\mathrm{G}$ on cell surfaces at the electron microscopic level: a new method, theory and application. Journal of Histochemistry \& Cytochemistry 1977, 25 (11), 1187-1200.

52. Bayraktar, H.; You, C.-C.; Rotello, V. M.; Knapp, M. J., Facial control of nanoparticle binding to cytochrome c. Journal of the American Chemical Society 2007, 129 (10), 27322733. 
53. Hainfeld, J. F.; Powell, R. D., New frontiers in gold labeling. Journal of Histochemistry \& Cytochemistry 2000, 48 (4), 471-480.

54. Mahmoudi, M.; Lynch, I.; Ejtehadi, M. R.; Monopoli, M. P.; Bombelli, F. B.; Laurent, S., Protein- nanoparticle interactions: opportunities and challenges. Chemical reviews 2011, $111(9), 5610-5637$.

55. Huh, Y.-M.; Jun, Y.-w.; Song, H.-T.; Kim, S.; Choi, J.-s.; Lee, J.-H.; Yoon, S.; Kim, K.-S.; Shin, J.-S.; Suh, J.-S., In vivo magnetic resonance detection of cancer by using multifunctional magnetic nanocrystals. Journal of the American Chemical Society 2005, 127 (35), 12387-12391.

56. Aubin-Tam, M.-E.; Hamad-Schifferli, K., Gold nanoparticle-cytochrome c complexes: the effect of nanoparticle ligand charge on protein structure. Langmuir 2005, 21 (26), 1208012084 .

57. Bagalkot, V.; Zhang, L.; Levy-Nissenbaum, E.; Jon, S.; Kantoff, P. W.; Langer, R.; Farokhzad, O. C., Quantum dot-aptamer conjugates for synchronous cancer imaging, therapy, and sensing of drug delivery based on bi-fluorescence resonance energy transfer. Nano letters 2007, 7 (10), 3065-3070.

58. (a) Lynch, I.; Dawson, K. A., Protein-nanoparticle interactions. Nano today 2008, 3 (1), 40-47; (b) Lundqvist, M.; Stigler, J.; Elia, G.; Lynch, I.; Cedervall, T.; Dawson, K. A., Nanoparticle size and surface properties determine the protein corona with possible implications for biological impacts. Proceedings of the National Academy of Sciences 2008, 105 (38), 14265-14270.

59. Lacerda, S. H. D. P.; Park, J. J.; Meuse, C.; Pristinski, D.; Becker, M. L.; Karim, A.; Douglas, J. F., Interaction of gold nanoparticles with common human blood proteins. ACS nano 2009, 4 (1), 365-379.

60. Cedervall, T.; Lynch, I.; Lindman, S.; Berggård, T.; Thulin, E.; Nilsson, H.; Dawson, K. A.; Linse, S., Understanding the nanoparticle-protein corona using methods to quantify exchange rates and affinities of proteins for nanoparticles. Proceedings of the National Academy of Sciences 2007, 104 (7), 2050-2055.

61. Klein, J., Probing the interactions of proteins and nanoparticles. Proceedings of the National Academy of Sciences 2007, 104 (7), 2029-2030.

62. Cox, M. C.; Barnham, K. J.; Frenkiel, T. A.; Hoeschele, J. D.; Mason, A. B.; He, Q.Y.; Woodworth, R. C.; Sadler, P., Identification of platination sites on human serum transferrin using 13C and 15N NMR spectroscopy. JBIC Journal of Biological Inorganic Chemistry 1999, 4 (5), 621-631.

63. 安德义; 苏计国; 李春华; 李敬源, Computational studies on the interactions of nanomaterials with proteins and their impacts. Chinese Physics B 2015, 12, 002.

64. Schulze, C.; Schulze, C.; Kroll, A.; Schulze, C.; Kroll, A.; Lehr, C.-M.; Schäfer, U. F.; Becker, K.; Schnekenburger, J.; Schulze Isfort, C., Not ready to use-overcoming pitfalls when dispersing nanoparticles in physiological media. Nanotoxicology 2008, 2 (2), 51-61.

65. Monopoli, M. P.; Walczyk, D.; Campbell, A.; Elia, G.; Lynch, I.; Baldelli Bombelli, F.; Dawson, K. A., Physical- chemical aspects of protein corona: relevance to in vitro and in vivo biological impacts of nanoparticles. Journal of the American Chemical Society 2011, 133 (8), 2525-2534.

66. Buijs, J.; Vera, C. C.; Ayala, E.; Steensma, E.; Håkansson, P.; Oscarsson, S., Conformational stability of adsorbed insulin studied with mass spectrometry and hydrogen exchange. Analytical chemistry 1999, 71 (15), 3219-3225. 
67. Nakanishi, K.; Sakiyama, T.; Imamura, K., On the adsorption of proteins on solid surfaces, a common but very complicated phenomenon. Journal of Bioscience and Bioengineering 2001, 91 (3), 233-244.

68. Yu, C. H.; Al-Saadi, A.; Shih, S.-J.; Qiu, L.; Tam, K. Y.; Tsang, S. C., Immobilization of BSA on silica-coated magnetic iron oxide nanoparticle. The Journal of Physical Chemistry $C$ 2008, 113 (2), 537-543.

69. Mora, M. F.; Wehmeyer, J. L.; Synowicki, R.; Garcia, C. D., Investigating protein adsorption via spectroscopic ellipsometry. In Biological Interactions on Materials Surfaces, Springer: 2009; pp 19-41.

70. Ehrenberg, M. S.; Friedman, A. E.; Finkelstein, J. N.; Oberdörster, G.; McGrath, J. L., The influence of protein adsorption on nanoparticle association with cultured endothelial cells. Biomaterials 2009, 30 (4), 603-610.

71. Chanteau, B.; Fresnais, J.; Berret, J.-F., Electrosteric enhanced stability of functional sub-10 nm cerium and iron oxide particles in cell culture medium. Langmuir 2009, 25 (16), 9064-9070.

72. Arima, Y.; Iwata, H., Effects of surface functional groups on protein adsorption and subsequent cell adhesion using self-assembled monolayers. Journal of Materials Chemistry 2007, 17 (38), 4079-4087.

73. Deng, Z. J.; Liang, M.; Monteiro, M.; Toth, I.; Minchin, R. F., Nanoparticle-induced unfolding of fibrinogen promotes Mac-1 receptor activation and inflammation. Nature nanotechnology 2011, 6 (1), 39-44.

74. Wangoo, N.; Suri, C. R.; Shekhawat, G., Interaction of gold nanoparticles with protein: a spectroscopic study to monitor protein conformational changes. Applied Physics Letters 2008, 92 (13), 133104.

75. Gole, A.; Dash, C.; Soman, C.; Sainkar, S.; Rao, M.; Sastry, M., On the preparation, characterization, and enzymatic activity of fungal protease-gold colloid bioconjugates.

Bioconjugate chemistry 2001, 12 (5), 684-690.

76. Huang, Y.-F.; Huang, C.-C.; Chang, H.-T., Exploring the activity and specificity of gold nanoparticle-bound trypsin by capillary electrophoresis with laser-induced fluorescence detection. Langmuir 2003, 19 (18), 7498-7502.

77. Zhang, Y.; He, P.; Hu, N., Horseradish peroxidase immobilized in TiO 2 nanoparticle films on pyrolytic graphite electrodes: direct electrochemistry and bioelectrocatalysis. Electrochimica Acta 2004, 49 (12), 1981-1988.

78. Calzolai, L.; Franchini, F.; Gilliland, D.; Rossi, F., Protein- nanoparticle interaction: identification of the ubiquitin- gold nanoparticle interaction site. Nano letters 2010, 10 (8), 3101-3105.

79. Kubiak, K.; Mulheran, P. A., Molecular dynamics simulations of hen egg white lysozyme adsorption at a charged solid surface. The Journal of Physical Chemistry B 2009, 113 (36), 12189-12200.

80. Nguyen, H. H.; Park, J.; Kang, S.; Kim, M., Surface plasmon resonance: a versatile technique for biosensor applications. Sensors 2015, 15 (5), 10481-10510.

81. Lee, O.-S.; Schatz, G. C., Interaction between DNAs on a gold surface. The Journal of Physical Chemistry C 2009, 113 (36), 15941-15947.

82. Tsai, W. B.; Grunkemeier, J. M.; McFarland, C. D.; Horbett, T. A., Platelet adhesion to polystyrene-based surfaces preadsorbed with plasmas selectively depleted in fibrinogen, 
fibronectin, vitronectin, or von Willebrand's factor. Journal of biomedical materials research 2002, 60 (3), 348-359.

83. Xie, Y.; Liu, M.; Zhou, J., Molecular dynamics simulations of peptide adsorption on self-assembled monolayers. Applied Surface Science 2012, 258 (20), 8153-8159.

84. Cho, E. C.; Xie, J.; Wurm, P. A.; Xia, Y., Understanding the role of surface charges in cellular adsorption versus internalization by selectively removing gold nanoparticles on the cell surface with a I2/KI etchant. Nano letters 2009, 9 (3), 1080-1084.

85. Lin, J.; Zhang, H.; Chen, Z.; Zheng, Y., Penetration of lipid membranes by gold nanoparticles: insights into cellular uptake, cytotoxicity, and their relationship. ACS nano 2010, 4 (9), 5421-5429.

86. Lin, J.-Q.; Zheng, Y.-G.; Zhang, H.-W.; Chen, Z., A simulation study on nanoscale holes generated by gold nanoparticles on negative lipid bilayers. Langmuir 2011, 27 (13), 8323-8332.

87. Verde, A. V.; Acres, J. M.; Maranas, J. K., Investigating the specificity of peptide adsorption on gold using molecular dynamics simulations. Biomacromolecules 2009, 10 (8), 2118-2128.

88. Raffaini, G.; Ganazzoli, F., Protein adsorption on a hydrophobic surface: a molecular dynamics study of lysozyme on graphite. Langmuir 2009, 26 (8), 5679-5689.

89. Zhou, J.; Zheng, J.; Jiang, S., Molecular simulation studies of the orientation and conformation of cytochrome c adsorbed on self-assembled monolayers. The Journal of Physical Chemistry B 2004, 108 (45), 17418-17424.

90. Sun, Y.; Latour, R. A., Comparison of implicit solvent models for the simulation of protein-surface interactions. Journal of computational chemistry 2006, 27 (16), 1908-1922. 91. Collier, G.; Vellore, N. A.; Yancey, J. A.; Stuart, S. J.; Latour, R. A., Comparison between empirical protein force fields for the simulation of the adsorption behavior of structured LK peptides on functionalized surfaces. Biointerphases 2012, 7 (1), 24.

92. Biswas, P. K.; Vellore, N. A.; Yancey, J. A.; Kucukkal, T. G.; Collier, G.; Brooks, B. R.; Stuart, S. J.; Latour, R. A., Simulation of multiphase systems utilizing independent force fields to control intraphase and interphase behavior. Journal of computational chemistry 2012, 33 (16), 1458-1466.

93. Wei, Y.; Latour, R. A., Correlation between desorption force measured by atomic force microscopy and adsorption free energy measured by surface plasmon resonance spectroscopy for peptide- surface interactions. Langmuir 2010, 26 (24), 18852-18861. 94. Heinz, H.; Jha, K. C.; Luettmer-Strathmann, J.; Farmer, B. L.; Naik, R. R., Polarization at metal-biomolecular interfaces in solution. Journal of The Royal Society Interface 2010, 20100318.

95. Iori, F.; Corni, S., Including image charge effects in the molecular dynamics simulations of molecules on metal surfaces. Journal of computational chemistry 2008, 29 (10), 1656-1666.

96. Hoefling, M.; Monti, S.; Corni, S.; Gottschalk, K. E., Interaction of $\beta$-sheet folds with a gold surface. PloS one 2011, 6 (6), e20925.

97. Kohn, W.; Sham, L. J., Self-consistent equations including exchange and correlation effects. Physical review 1965, 140 (4A), A1133.

98. Wright, L. B.; Rodger, P. M.; Corni, S.; Walsh, T. R., GolP-CHARMM: Firstprinciples based force fields for the interaction of proteins with $\mathrm{Au}$ (111) and $\mathrm{Au}(100)$. Journal of chemical theory and computation 2013, 9 (3), 1616-1630. 
99. Cicero, G.; Calzolari, A.; Corni, S.; Catellani, A., Anomalous wetting layer at the Au (111) surface. The Journal of Physical Chemistry Letters 2011, 2 (20), 2582-2586.

100. Grochola, G.; Russo, S. P.; Snook, I. K., On fitting a gold embedded atom method potential using the force matching method. The Journal of chemical physics 2005, 123 (20), 204719.

101. Plimpton, S., Fast parallel algorithms for short-range molecular dynamics. Journal of computational physics 1995, 117 (1), 1-19.

102. Wei, T.; Carignano, M. A.; Szleifer, I., Lysozyme adsorption on polyethylene surfaces: why are long simulations needed? Langmuir 2011, 27 (19), 12074-12081.

103. (a) Ghorai, P. K.; Glotzer, S. C., Molecular dynamics simulation study of selfassembled monolayers of alkanethiol surfactants on spherical gold nanoparticles. The Journal of Physical Chemistry C 2007, 111 (43), 15857-15862; (b) Alexiadis, O.; Harmandaris, V. A.; Mavrantzas, V. G.; Site, L. D., Atomistic simulation of alkanethiol self-assembled monolayers on different metal surfaces via a quantum, first-principles parametrization of the sulfur-metal interaction. The Journal of Physical Chemistry C 2007, 111 (17), 6380-6391.

104. (a) Zheng, J.; Li, L.; Tsao, H.-K.; Sheng, Y.-J.; Chen, S.; Jiang, S., Strong repulsive forces between protein and oligo (ethylene glycol) self-assembled monolayers: A molecular simulation study. Biophysical journal 2005, 89 (1), 158-166; (b) Zheng, J.; Li, L.; Chen, S.; Jiang, S., Molecular simulation study of water interactions with oligo (ethylene glycol)terminated alkanethiol self-assembled monolayers. Langmuir 2004, 20 (20), 8931-8938. 105. Haile, J., Molecular dynamics simulation. Wiley, New York: 1992; Vol. 18.

106. Allen, M. P., Introduction to molecular dynamics simulation. Computational soft matter: from synthetic polymers to proteins 2004, 23, 1-28.

107. Petrenko, R.; Meller, J., Molecular dynamics. eLS 2010.

108. Mandell, M., On the properties of a periodic fluid. Journal of Statistical Physics 1976, 15 (4), 299-305.

109. Hong Enriquez, R., Molecular Simulations of Metal-based Proteins: from Catalysis to Ion Transport. 2009.

110. Steinbach, P. J.; Brooks, B. R., New spherical-cutoff methods for long-range forces in macromolecular simulation. Journal of computational chemistry 1994, 15 (7), 667-683.

111. Gibbs, J. W., Elementary principles in statistical mechanics. Courier Corporation: 2014.

112. Barriga, J.; Coto, B.; Fernandez, B., Molecular dynamics study of optimal packing structure of OTS self-assembled monolayers on SiO 2 surfaces. Tribology International 2007, 40 (6), 960-966.

113. Humphrey, W.; Dalke, A.; Schulten, K., VMD: visual molecular dynamics. Journal of molecular graphics 1996, 14 (1), 33-38.

114. Li, J., AtomEye: an efficient atomistic configuration viewer. Modelling and Simulation in Materials Science and Engineering 2003, 11 (2), 173.

115. Neumann, J. Molecular dynamics simulations of protein-protein interactions and $\mathrm{THz}$ driving of molecular rotors on gold. lmu, 2011.

116. Vanommeslaeghe, K.; Hatcher, E.; Acharya, C.; Kundu, S.; Zhong, S.; Shim, J.; Darian, E.; Guvench, O.; Lopes, P.; Vorobyov, I., CHARMM general force field: A force field for drug-like molecules compatible with the CHARMM all-atom additive biological force fields. Journal of computational chemistry 2010, 31 (4), 671-690. 
117. Jones, J. E. In On the determination of molecular fields. II. From the equation of state of a gas, Proceedings of the Royal Society of London A: Mathematical, Physical and Engineering Sciences, The Royal Society: 1924; pp 463-477.

118. Jorgensen, W. L.; Maxwell, D. S.; Tirado-Rives, J., Development and testing of the OPLS all-atom force field on conformational energetics and properties of organic liquids. Journal of the American Chemical Society 1996, 118 (45), 11225-11236.

119. Girifalco, L. A.; Weizer, V. G., Application of the Morse potential function to cubic metals. Physical Review 1959, 114 (3), 687.

120. Henz, B. J.; Hawa, T.; Zachariah, M. R., Mechano-chemical stability of gold nanoparticles coated with alkanethiolate SAMs. Langmuir 2008, 24 (3), 773-783.

121. Gagner, J. E.; Lopez, M. D.; Dordick, J. S.; Siegel, R. W., Effect of gold nanoparticle morphology on adsorbed protein structure and function. Biomaterials 2011, 32 (29), 72417252 .

122. (a) Chen, S.; Zheng, J.; Li, L.; Jiang, S., Strong resistance of phosphorylcholine selfassembled monolayers to protein adsorption: insights into nonfouling properties of zwitterionic materials. Journal of the American Chemical Society 2005, 127 (41), 1447314478; (b) Harder, P.; Grunze, M.; Dahint, R.; Whitesides, G.; Laibinis, P., Molecular conformation in oligo (ethylene glycol)-terminated self-assembled monolayers on gold and silver surfaces determines their ability to resist protein adsorption. The Journal of Physical Chemistry B 1998, 102 (2), 426-436.

123. Camillone III, N.; Chidsey, C. E.; Liu, G. y.; Scoles, G., Superlattice structure at the surface of a monolayer of octadecanethiol self-assembled on Au (111). The Journal of chemical physics 1993, 98 (4), 3503-3511.

124. Vemparala, S.; Karki, B. B.; Kalia, R. K.; Nakano, A.; Vashishta, P., Large-scale molecular dynamics simulations of alkanethiol self-assembled monolayers. The Journal of chemical physics 2004, 121 (9), 4323-4330.

125. (a) Hautman, J.; Bareman, J. P.; Mar, W.; Klein, M. L., Molecular dynamics investigations of self-assembled monolayers. Journal of the Chemical Society, Faraday Transactions 1991, 87 (13), 2031-2037; (b) Fenter, P.; Eberhardt, A.; Liang, K.; Eisenberger, P., Epitaxy and chainlength dependent strain in self-assembled monolayers. The Journal of chemical physics 1997, 106 (4), 1600-1608; (c) Caldwell, W. B.; Campbell, D. J.; Chen, K.; Herr, B. R.; Mirkin, C. A.; Malik, A.; Durbin, M.; Dutta, P.; Huang, K., A highly ordered self-assembled monolayer film of an azobenzenealkanethiol on Au (111): Electrochemical properties and structural characterization by synchrotron in-plane X-ray diffraction, atomic force microscopy, and surface-enhanced Raman spectroscopy. Journal of the American Chemical Society 1995, 117 (22), 6071-6082.

126. Singh-Miller, N. E.; Marzari, N., Surface energies, work functions, and surface relaxations of low-index metallic surfaces from first principles. Physical Review B 2009, 80 (23), 235407.

127. Ostuni, E.; Grzybowski, B. A.; Mrksich, M.; Roberts, C. S.; Whitesides, G. M., Adsorption of proteins to hydrophobic sites on mixed self-assembled monolayers. Langmuir 2003, 19 (5), 1861-1872.

128. Nawrocki, G.; Cieplak, M., Aqueous Amino Acids and Proteins Near the Surface of Gold in Hydrophilic and Hydrophobic Force Fields. The Journal of Physical Chemistry C 2014, 118 (24), 12929-12943. 
129. Lopez, H.; Lobaskin, V., Coarse-grained model of adsorption of blood plasma proteins onto nanoparticles. The Journal of chemical physics 2015, 143 (24), 243138.

130. Xie, Y.; Zhou, J.; Jiang, S., Parallel tempering Monte Carlo simulations of lysozyme orientation on charged surfaces. The Journal of chemical physics 2010, 132 (6), 065101. 131. Thompson, D.; Sikora, M.; Szymczak, P.; Cieplak, M., A multi-scale molecular dynamics study of the assembly of micron-size supraparticles from $30 \mathrm{~nm}$ alkyl-coated nanoparticles. Physical Chemistry Chemical Physics 2013, 15 (21), 8132-8143. 